\title{
第一过渡系金属手性双核催化剂和相应的非线性效应研究进展
}

\author{
杨艳王立颖罗君朱育林* \\ (华南师范大学化学与环境学院 广州 510006)
}

\begin{abstract}
摘要 第一过渡系金属(3d 金属)手性双核配合物的研究已渗透到不对称催化、生物酶模拟、功能超分子材料、医药等 众多领域, 是当前非常具有吸引力和应用前景的研究热点. 相比传统的单核金属有机催化剂, 第一过渡系金属手性双 核配合物因催化活性中心的增加以及双核间的协同作用，在许多对映选择性反应中表现出优异的性能. 综述了近 10 年 来, 多种第一过渡系金属 $(\mathrm{V}, \mathrm{Ti}, \mathrm{Mn}, \mathrm{Fe}, \mathrm{Co}, \mathrm{Ni}, \mathrm{Cu}, \mathrm{Zn}$ )手性双核催化剂在不对称反应中研究进展, 如不对称加成、动力 学拆分、选择性氧化、不对称聚合等反应. 同时, 对基于同-或异一手性双核配合物的非线性效应研究近况进行了简单 的探讨.
\end{abstract}

关键词 手性双核催化剂; 第一过渡系金属; 不对称催化; 非线性效应

\section{Research and Progress in Chiral Binuclear Catalyst of First Row Transition Metal and Corresponding Nonlinear Effect}

\author{
Yang, Yan Wang, Liying Luo, Jun Zhu, Yulin* \\ (School of Chemistry and Enviroment, South China Normal University, Guangzhou 510006)
}

\begin{abstract}
The study on chiral binuclear complexes of first row transition metal has been expended to correlated fields such as asymmetric catalysis, biomimetic, functional supramolecular material and medicine, and reaches a research hot point of vast attraction and potential. Contrasting with other traditional organic-metal catalysts, the chiral binuclear complexes which combine several chiral centers and cooperated binuclear often play preferable catalysis behavior in enantioselective reaction. This review covers the recent progress in chiral binuclear complexes of $\mathrm{V}, \mathrm{Ti}, \mathrm{Mn}, \mathrm{Fe}, \mathrm{Co}, \mathrm{Ni}, \mathrm{Cu}, \mathrm{Zn}$ applied in asymmetric reactions involving asymmetric addition, kinetic resolution, selective oxidation, asymmetric polymerization, as well as the mechanistic investigations. Some studies on the nonlinear effect along with the dimer catalysts are also discussed. The challenges and perspectives are given at the end of this article.
\end{abstract}

Keywords chiral binuclear complexes; first row transition metal; asymmetric catalysis; nonlinear effect

金属有机催化剂种类丰富，结构复杂多样. 因其具 有高催化活性与选择性, 在手性合成中占据着重要地 位. 第一过渡系金属元素, 又被称为 $3 \mathrm{~d}$ 金属或轻过渡金 属, 它们廉价、环境友好且具备较大的生物相关性 ${ }^{[1]}$. 与 传统单金属催化剂相比，第一过渡系金属手性双核配合 物因催化活性中心增加 ${ }^{[2]}$ 以及双核间的协同作用 ${ }^{[3]}$, 往 往表现出更高的催化活性和较理想的对映选择性 ${ }^{[4]}$, 特 别是对于同时需要双活化的催化反应. 两金属核相互靠 近, 也使参与反应的两组分之间结合的熵值降低, 溶剂 间重排能垒最小化 ${ }^{[5]}$. 分子质量的增加也有利于催化剂 的分离回收利用 ${ }^{[6]}$. 吴毓林等 ${ }^{[7]}$ 将双金属催化剂作为催 化剂设计领域上的新趋向，在其专著中予以介绍.

吴毓林等同时还介绍了不对称催化反应中的非线
性效应 ${ }^{[7]}$, 即产物 $e e$ 值与手性助剂或催化剂的 $e e$ 值偏离 线性的比例关系 ${ }^{[8]}$ (图 1). Finn 和 Sharpless 等 ${ }^{[9]}$ 认为异手 性配体二聚形成高稳定性低催化活性的双核配合物，消 除了催化体系混杂的少量外消旋体, 从而增大了实际参 与催化的手性配体的对映纯度. Noyori 等 ${ }^{[10]}$ 也认为同或异-手性二聚配合物是不对称催化中非线性效应现象 的根源. 正的非线性效应又称作手性放大 ${ }^{[11]}$, 在手性合 成应用上有着巨大的发展潜力.

张占金 ${ }^{[12]}$ 和高月华等 ${ }^{[13]}$ 分别介绍了手性双核催化 剂在不对称反应中的应用，但该两篇文献同样都将篇幅 集中在了贵重金属铑、铂上. 第一过渡系手性双核配合 物在生物酶模拟 ${ }^{[14]}$, 超分子构建 ${ }^{[15]}$, 磁光电材料 ${ }^{[16]}$, 分 子识别 ${ }^{[17]}$ 以及手性孔隙多功能材料 ${ }^{[18]}$ 等方面的应用成

*E-mail: yulinzhu2002@aliyun.com

Received January 9, 2013; revised February 6, 2013; published online February 18, 2013. 


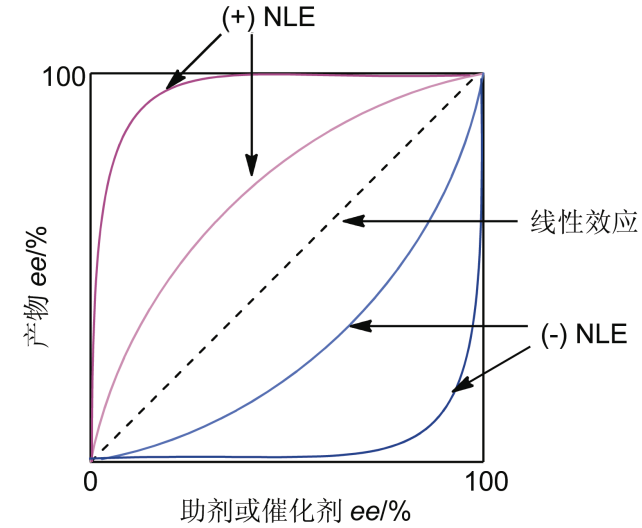

图 1 不对称催化中的非线性效应(NLE)

Figure 1 Nonliear effect relationship in enantioselective catalysis

果诸多. 本文归纳综述了第一过渡系金属手性双核催化 剂在不对称加成、动力学拆分、选择性氧化和对映选择 性聚合等反应上的研究应用进展和催化机理，同时还对 由同-或异-手性双核配合物引起的非线性相关研究作 了简单探讨.

\section{1 第一过渡系金属手性双核配合物催化剂}

为数众多的天然双核酶 ${ }^{[1]}$ 协同催化所表现出的高催 化效能与高选择性引发了众多学者的关注. 以修饰型 $(R, R)$-Salen 配体-、天然氨基酸衍生配体-、联荟衍生物 配体 ${ }^{[19]}$-金属配合物为代表的第一过渡系金属手性双核 催化剂在许多不对称反应上的催化研究取得了巨大的 成功.

\section{1 不对称加成催化剂}

对 $\mathrm{sp}^{2}$ 碳潜手性中心进行面选择性加成, 是构建手 性中心重要策略. Belokon 等在用 $\mathrm{Salen}-\mathrm{TiCl}_{2}$ 催化醛与 $\mathrm{Me}_{3} \mathrm{SiCN}$ 的不对称加成反应中, 发现 “ $\mathrm{H}_{2} \mathrm{O}$ ” 氧桥双核 钛配合物表现优越的催化性能 ${ }^{[20]}$, 相邻的两个 $\mathrm{Ti}$ 核一 个活化亲核的腈基另一个活化亲电的羰基 ${ }^{[21]}$. 此类研 究拉开了手性双核配合物在催化不对称反应方面研究 的序幕.

Belokon 等 ${ }^{[22]}$ 同时还报道了单核与双核催化剂在溶 液中存在一个基于浓度的动态平衡. 丁奎岭小组 ${ }^{[23]}$ 为 提高双核比例, 合成了以 $\left[\mathrm{Ti}_{2}(\mathbf{L 1})(\mu-\mathrm{O})_{2}\right]$ 为代表的协同 性联-Salen 型双钛催化剂(Chart 1), 催化醛与硅氧的不 对称加成, 室温下以 $0.01 \mathrm{~mol} \%$ 的超低催化负载, 较短 时间内反应最高可达到 $99 \%$ 收率和 $96 \%$ 的 $e e$ 值. 作者同 期设计的联基过长或 $\mathrm{Ti}$ 未处在可协同作用的位置的催 化剂，活性及选择性都不理想.

不对称 Henry 反应是形成不对称 $\mathrm{C}-\mathrm{C}$ 键的重要手

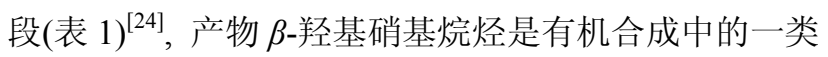

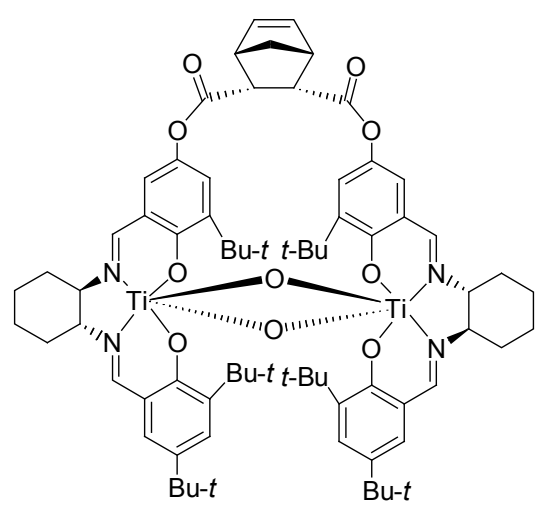

$\left[\mathrm{Ti}_{2}(\mathrm{~L} 1)(\mu-\mathrm{O})_{2}\right]$

\section{Chart 1}

重要中间体 ${ }^{[25]}$. Jammi 等 ${ }^{[26]}$ 合成了亚胺 $(S)$-氨基醇的双 核配合物 $\left[\mathrm{Cu}_{2}^{\mathrm{II}}(\mathbf{L 2})_{2}\right]$ (Chart 2), 作为 Brønsted 碱和 Lewis 酸双功能催化剂应用于 Henry 反应(表 1, Entry 1). 饱和胺对这类反应的催化性能要高于不饱和的亚胺. Shi 等 ${ }^{[27]}$ 将还原 Salen 配体与 $\mathrm{Cu}(\mathrm{OAc})_{2}$ 在 $\mathrm{MeOH}$ 中按 $1 ： 1.2$ 配位, 分离得到酚桥 $\left[\mathrm{Cu}_{2}^{\mathrm{II}}(\mathbf{L 3})_{2}\right]$, 采用原位法催 化 Henry 反应，对映选择性较高(表 1, Entries 2,3). 半冠 型 Trost 叔胺双功能催化剂 $\left[\mathrm{Zn}_{2}(\mathbf{L} 4)(\mathrm{Et})\right]$, 在 $-35 \sim 4{ }^{\circ} \mathrm{C}$ 低温下可有效催化一系列的包括 Henry 反应 ${ }^{[28]}$ 在内的 $\mathrm{C}-\mathrm{C}$ 偶合生成手性醇的反应(表 1 , Entries 4, 5) ${ }^{[29]}$.
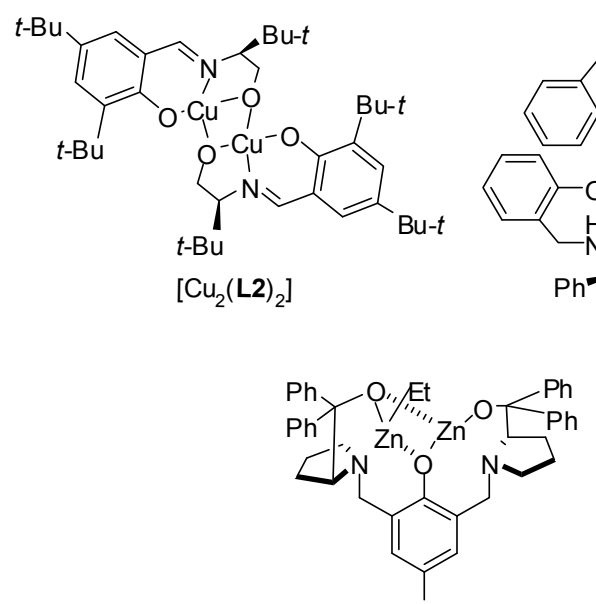

$\left[\mathrm{Zn}_{2}(\mathrm{~L} 4) \mathrm{Et}\right]$

\section{Chart 2}

Kureshy 等 ${ }^{[6]}$ 在通过 Henry 反应合成 $(R)$-苯肾上腺素 时，设计了大环双 Salen 配体 $\mathrm{H}_{4} \mathbf{L} 5 \mathbf{a}, \mathrm{H}_{4} \mathbf{L} \mathbf{b}$, 用 $\mathrm{NaBF}_{4}$ 还原处理得 $\mathrm{H}_{4} \mathbf{L 5 c}, \mathrm{H}_{4} \mathbf{L 5 d}$ (Eq. 1), 与 $\mathrm{Cu}(\mathrm{OAc})_{2}$ 原位催 化 Henry 反应，结果含二苯基二胺单元的 $\left[\mathrm{Cu}^{\mathrm{II}}{ }_{2}(\mathbf{L 5 d})\right]$ 催 化能力比相应含环己基二胺单元的 $\left[\mathrm{Cu}^{\mathrm{II}}{ }_{2}(\mathbf{L 5 c})\right]$ 强. 在对 其机理的研究中, Kureshy 指出, 二胺上的苯基与芳香醛 上的苯基通过 $\pi-\pi$ 堆积相互作用，使生成 $(R)$-构型醇 


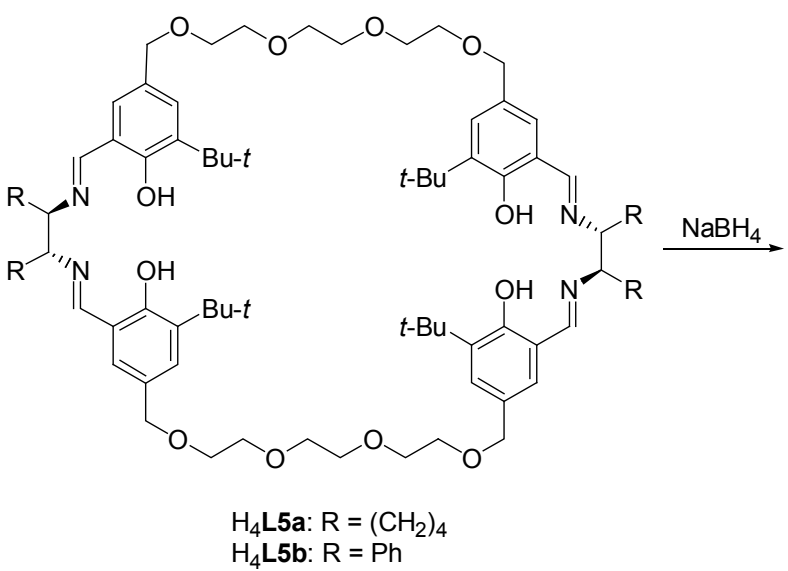

的空间障碍小. 多次回收利用催化剂效能未衰减(表 1 , Entries 6〜9).

表 1 不对称 Henry 反应

Table 1 Asymmetric Henry reaction

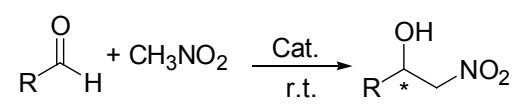

\begin{tabular}{|c|c|c|c|c|c|c|}
\hline Entry & $\mathrm{R}$ & Cat. & Sol. & $\begin{array}{l}\text { Loading/ } \\
\text { mol\% }\end{array}$ & Yield $/ \%$ & $\begin{array}{c}e e / \% \\
\text { (Conf.) }\end{array}$ \\
\hline 1 & $4-\mathrm{NO}_{2} \mathrm{C}_{6} \mathrm{H}_{4}$ & $\mathrm{Cu}_{2}-\mathbf{L} \mathbf{2}$ & $\mathrm{CH}_{2} \mathrm{Cl}_{2}$ & 2.5 & 90 & $32(S)$ \\
\hline 2 & $2-\mathrm{ClC}_{6} \mathrm{H}_{4}$ & $\mathrm{Cu}_{2}-\mathbf{L} \mathbf{3}$ & THF & 5 & 75 & $67(R)$ \\
\hline 3 & 1-Naphthyl & $\mathrm{Cu}_{2}-\mathbf{L} \mathbf{3}$ & THF & 5 & 30 & $83(R)$ \\
\hline 4 & $\mathrm{Ph}$ & $\mathrm{Zn}_{2}-\mathbf{L} 4$ & THF & 5 & 85 & $91(R)$ \\
\hline 5 & $\mathrm{CH}\left(\mathrm{C}_{2} \mathrm{H}_{5}\right)_{2}$ & $\mathrm{Zn}_{2}-\mathbf{L 4}$ & THF & 5 & 90 & $92(R)$ \\
\hline 6 & $2-\mathrm{ClC}_{6} \mathrm{H}_{4}$ & $\mathrm{Cu}_{2}-\mathbf{L 5 c}$ & THF & 15 & 64 & $56(R)$ \\
\hline 7 & 2- $\mathrm{ClC}_{6} \mathrm{H}_{4}$ & $\mathrm{Cu}_{2}-\mathbf{L 5 d}$ & THF & 15 & 94 & $96(R)$ \\
\hline 8 & $4-\mathrm{NO}_{2} \mathrm{C}_{6} \mathrm{H}_{4}$ & $\mathrm{Cu}_{2}-\mathbf{L} \mathbf{5 d}$ & THF & 5 & 90 & $89(R)$ \\
\hline 9 & 1-Naphthyl & $\mathrm{Cu}_{2}-\mathbf{L 5 d}$ & THF & 5 & 92 & $88(R)$ \\
\hline
\end{tabular}

手性喹啉联荎膦配体 $(R)-\mathbf{L 6}$ 与 $\mathrm{CuBr}$ 催化端基炔, 醛和二胺的多组分反应可得到高达 $96 \%$ 的 $e e$ 值(Eq. 2), 同时存在一个正的非线性效应 ${ }^{[30]}$. 鉴于异手性 $\left[\mathrm{Cu}_{2}^{\mathrm{I}}\right.$ $\left.(\mu-\mathrm{Br})_{2}((R)-\mathbf{L 6})((S)-\mathbf{L} 6)\right]$ 的反应活性较低, 同时分离出 了同手性双核 $\left[\mathrm{Cu}_{2}^{\mathrm{I}}(\mu-\mathrm{Br})_{2}((R)-\mathbf{L 6})_{2}\right]$ 单晶 ${ }^{[31]}$, 说明这种 非线性效应很可能是由于同手性配体优先生成高催化 活性的 $\left[\mathrm{Cu}_{2}^{\mathrm{I}}(\mu-\mathrm{Br})_{2}((R)-\mathbf{L} \mathbf{6})_{2}\right]$, 从而使体系的对映选择 性升高引起的.
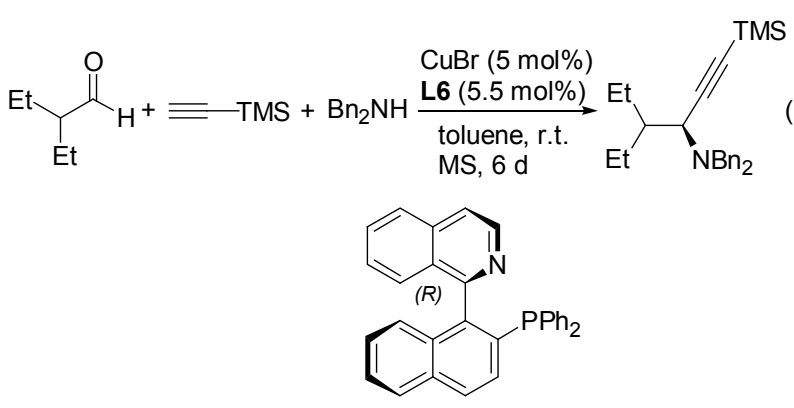

(R)-L6

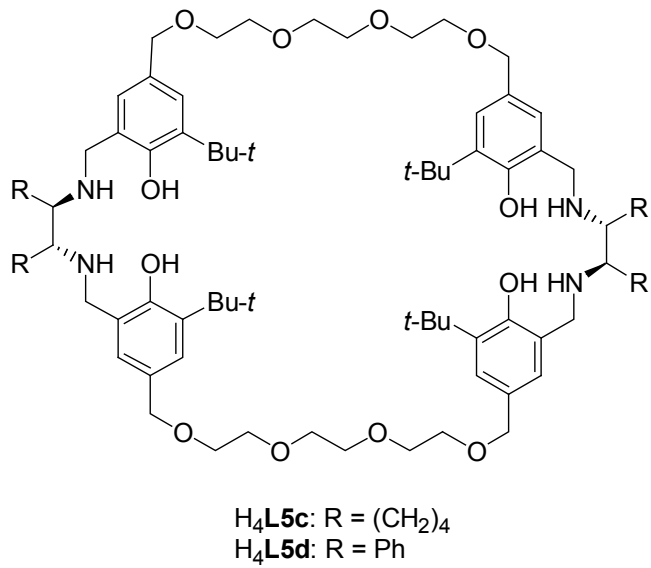

(1)

Sekar 小组 ${ }^{[32]}$ 旨在寻求经济环保、制备方法简单的 手性催化剂，他们开创性地将 Salen-Fe(III)催化剂应于 催化醛的不对称磷酸酯加成, 并分离出起催化活性的 $\left[\mathrm{Fe}_{2}^{\mathrm{III}}(\mathbf{L} 7)_{2} \mathrm{Cl}_{2}\right]$, 收率最高达 98\%, ee 值也可高达 64\%. 催化过程中, 易离去的氯配体, 有助于亲电亲核双活化 过程的实现 (Scheme 1) ${ }^{[33]}$.
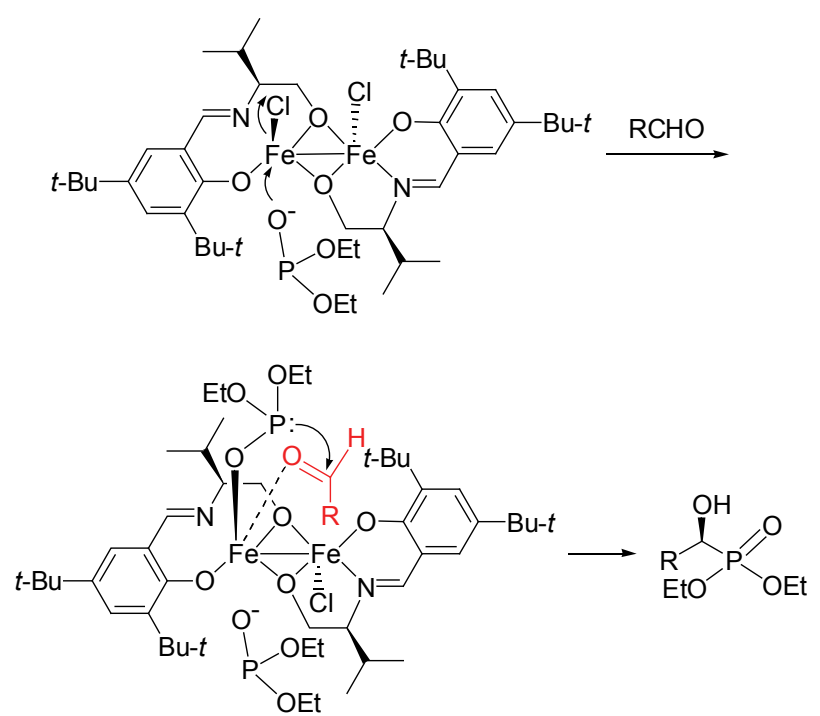

Scheme 1

含手性轴的联菱或联苯是不对称催化中最有效的 配体之一. 通常, $(S)-\mathrm{H}_{8}$-BINOL 往往比相应的 $(S)-$ BINOL 表现更高的对映选择性 ${ }^{[34]}$. 两者与 Ti $(\mathrm{O}-i-\mathrm{Pr})_{4}$ 一起 催化醛与烷基锌的不对称加成反应，不存在非线性效 应, 说明催化活性结构中仅含单一手性配体. 结合晶体 数据, Walsh 等 ${ }^{[35]}$ 结合晶体表征结果认为 $\left[\mathrm{Ti}_{2}^{\mathrm{IV}}\left((S)-\mathrm{H}_{8}-\right.\right.$ BINOL)(O-i-Pr) $)_{6}$ (Chart 3) 是催化活性物质. 稍过量的 $\mathrm{Ti}(\mathrm{O}-i-\mathrm{Pr})_{4}$ 是保证体系中此种活性结构占主导的必要条 件, 两者 $e e$ 值分别为 $98 \%$ 和 $92 \% . \mathrm{H}_{8}$-BINOL 中环已烷 平面稍大的二面角 $\left(7^{\circ}\right)$ 和空间需求很可能是对映选择性 高的原因 ${ }^{[36]}$. 


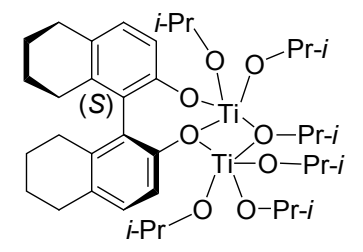

$\left[\mathrm{Ti}_{2}\left((S)-\mathrm{H}_{8}-\mathrm{BINOL}\right)(\mathrm{O}-i-\mathrm{Pr})_{6}\right]$

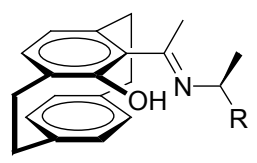

$\mathrm{R}=$ 环已基 $\quad\left(R_{\mathrm{P}}, \mathrm{S}\right)-\mathrm{HL} \mathbf{8 a}$ $\mathrm{R}=\mathrm{Ph} \quad\left(R_{\mathrm{P}}, \mathrm{S}\right)-\mathrm{HL} \mathbf{8 b}$

Chart 3

Brase 小组 ${ }^{[37]}$ 率先指出, 烷基锌与肉桂醛的不对称 1,4-共轭加成反应, 无需其它金属盐辅助, $\left(R_{\mathrm{p}}\right)$-对环芳亚 胺配体 $\left(R_{\mathrm{p}}, S\right)-\mathrm{HL8}$ 与烷基锌形成手性双核配合物 $\left[\mathrm{Zn}_{2}\left(\left(R_{\mathrm{p}}, S\right)-\mathbf{L 8}\right)_{2} \mathrm{Et}_{2}\right]$. 和催化烷基锌与醛的不对称加成 反应一样 ${ }^{[38]},\left(R_{\mathrm{p}}, S\right)-\mathrm{HLC}$ 在此反应中也表现负的线性效 应, 这可能是由于 $\left.\mathrm{Zn}_{2}\left(\left(S_{\mathrm{p}}, S\right)-\mathbf{L 8}\right)_{2} \mathrm{Et}_{2}\right]$ 催化性能高于 $\left[\mathrm{Zn}_{2}\left(\left(R_{\mathrm{p}}, S\right)-\mathbf{L 8}\right)_{2} \mathrm{Et}_{2}\right]$ 引起的. 结合晶体结构和 DFT 理论 计算结果, 作者假设 $(\mathrm{ZnL})_{2}$ 型结构的手性双核配合物是 自催化前体，通过形成协同的团簇或拉长锌与烷基之间 的距离, 促进反应进行 1,4-选择性加成.

Shibasaki 小组合成了 BINOL 或联苯-Salen 型双核 双功能催化剂 $\left[\mathrm{M}_{2}((R)-\mathbf{L 9})\right]$ 和 $\left[\mathrm{Ni}_{2}((R)-\mathbf{L 1 0})\right]$ (Chart 4). 一般外围 $\mathrm{O} 4$ 配位的金属充当 Brønsted 中心而内围 $\mathrm{N}_{2} \mathrm{O}_{2}$ 配位的 $\mathrm{Ni}$ 核起 Lewis 酸作用. 这类催化剂催化羰基的 $\alpha$ 位上的不对称加成缩合反应表现出非常高的催化性能.

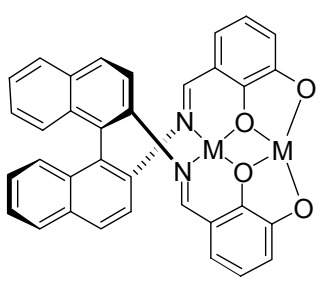

$\left[\mathrm{M}_{2}((R)-\mathrm{L} 9 \mathrm{a})\right]$

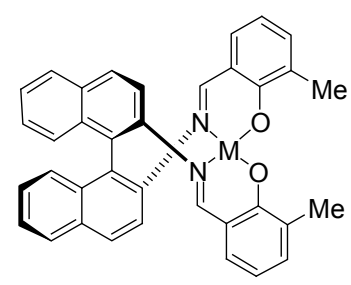

$[\mathrm{M}((R)-\mathrm{L} \mathbf{9 b})]$

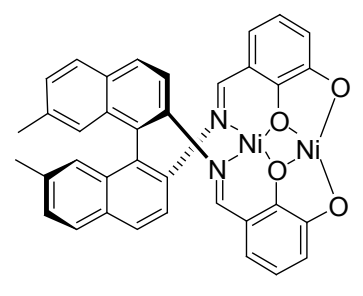

$\left[\mathrm{Ni}_{2}((R)-\mathrm{L} 10)\right]$

Chart 4

在催化 $\beta$-羰基磷酸酯与亚胺的不对称 Mannish 反应 时, Shibasaki 小组 ${ }^{[39]}$ 尝试了不同的金属核, $\mathrm{Cu}^{\mathrm{II}}-\mathrm{Sm}$ 或 $\mathrm{Ni}-\mathrm{Sm}$ 异双核以及 $\mathrm{Cu}^{\mathrm{I}}$ 双核的 $\left[\mathrm{M}_{2}((R)-\mathbf{L 9 a})\right]$ 配合物虽然 催化活性较高, 但基本上没有对映选择性, 而 $\left[\mathrm{Ni}_{2}((R)-\right.$ L9a)] 最高可表现高达 99\%的对映选择性和 $90 \%$ 的收率, $20: 1$ 的顺反式比值 (Eq. 3). 作者在将 $\left[\mathrm{Mn}_{2}^{\mathrm{III}}((R)-\right.$ L9a) $(\mathrm{OAc})_{2}$ ]成功应用到 3-取代吲哚与硝基烷烃的 1,4-
不对称加成后 ${ }^{[40]}$, 将反应底物扩展到 3-取代- $N$-代吲哚 酮和偶氮(Eq. 4 $)^{[41]}$. 改用 $(R)$-构型单核 $[\mathrm{Ni}((R)-\mathbf{L 9 b})]$ 作 催化剂, ee 值和活性基本上不变, 但产物为 $(S)$-构型产 物. 3 个 Boc 保护基非常容易移除, 生成手性 3-胺基吲 哚 ${ }^{[42]}$.

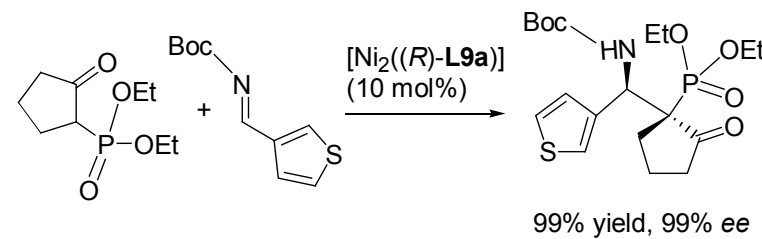
20:1 syn/tran

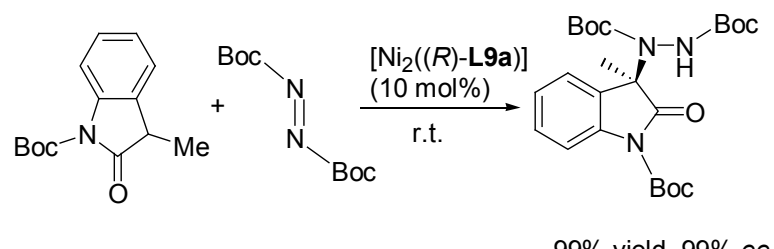

Shibasaki 小组 ${ }^{[43]}$ 在对映选择性全合成 caprezamycin $\mathrm{B}$ 尝试中, 认为 3-甲基戊二酸酐的溶剂解去对称化形 成手性单酯是关键转变. 而应用 $\left[\mathrm{Ni}_{2}((R)-\mathbf{L} 9 \mathbf{a})\right]$ 催化可达 $94 \%$ 的 ee 值和 $91 \%$ 的收率.

通过硝基烯烃类化合物的不对称 Micheal 加成可以 同时引入两个手性中心. 对 $\alpha$-羰基酰胺与硝基烯烃的 1,4-加成, 手性联萗配体 L9a 比联苯配体 L10 催化的对 映选择性与活性低(Scheme 2$),\left[\mathrm{Ni}_{2}((R)-\mathbf{L 1 0})\right]$ 与分子篎、 $1,1,1,3,3,3-$ 六氟异丙醇(HFIP)在 1,4 -二氧六环中, 催化 此反应对大部分芳香硝基烯烃底物呈中等以上的活性 及 $e e$ 值, 以及 $8.3: 1 \sim 20: 1$ 的顺反比 ${ }^{[44]}$. 加成产物通 过立体选择性的还原环化生成手性吡咯.

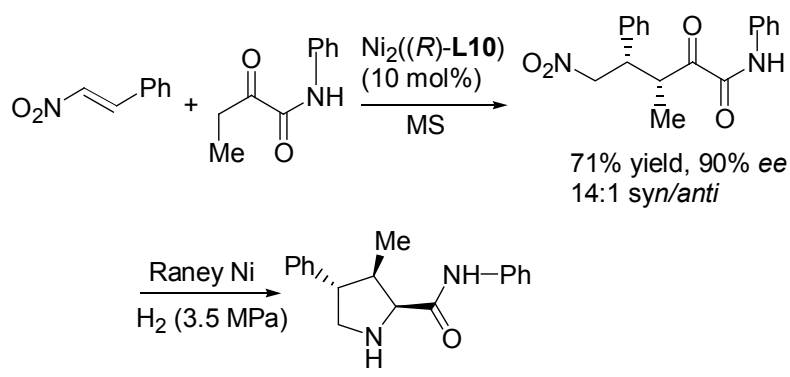

Scheme 2

Kleij 等用热液法合成了与 $(R)-\mathbf{L 1 0}$ 相似但联苯基上 无甲基取代的 L11 (Chart 5), 联苯轴的旋转使 $\left[\mathrm{Zn}_{2}(\mathbf{L 1 1})\right]$ 在 $(R)$-与 $(S)$ - 型之间存在一个动态平衡, 室温 下可作为微量的极性手性底物如手性酸的主体 ${ }^{[45]}$, 然 而过量的锌盐导致多核配合物生成，造成这种主体功能 失效 ${ }^{[46]}$. 


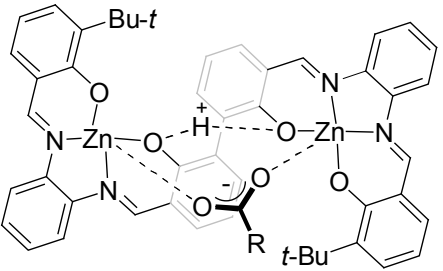

$\left[\mathrm{Ni}_{2}(\mathrm{~L} 11)(\mathrm{RCOOH})\right]$

\section{Chart 5}

\section{2 动力学拆分催化剂}

环氧化合物的水解动力学拆分 $(\mathrm{HKR})$ 被认为是最简 单有效普遍适用的获得纯手性端环氧的方法. Jacobsen 等发现一些手性 Salen-金属催化剂催化的反应如环氧水 解动力学拆分 (HKR), 对催化剂浓度表现出二阶适应关 系. 现普遍认为, 此反应经历了一个双核双活化过程 (Scheme 3) ${ }^{[47]}$.

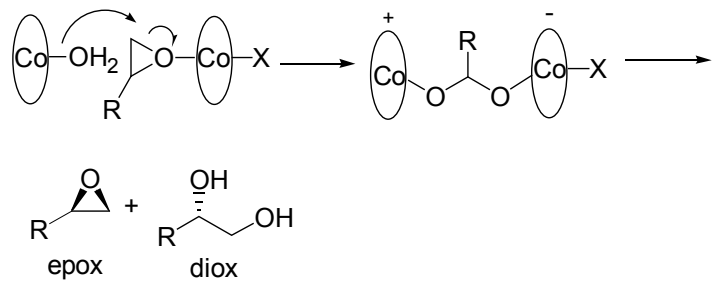

Scheme 3

双-Salen 型配体(Chart 6)的协同性双核配合物在这 类反应中得到了广泛的应用. 金属核面面相对，协同作 用较强, 稳定性高的杯芳烃型 $\left[\mathrm{Co}_{2}^{\mathrm{III}}(\mathrm{L2})(\mathrm{OTs})_{2}\right]$ 催化端 环氧的 HKR 对映选择性非常高(表 2, Entry 1), 但杯芳 烃单元使得催化剂的成本升高了许多 ${ }^{[48]}$.

Weck 和 Jones 等 ${ }^{[49]}$ 合成了以苯乙烯-4-低聚乙二醇Salen 配体为主链结构的可聚 Salen 配体, 聚合配合氧化
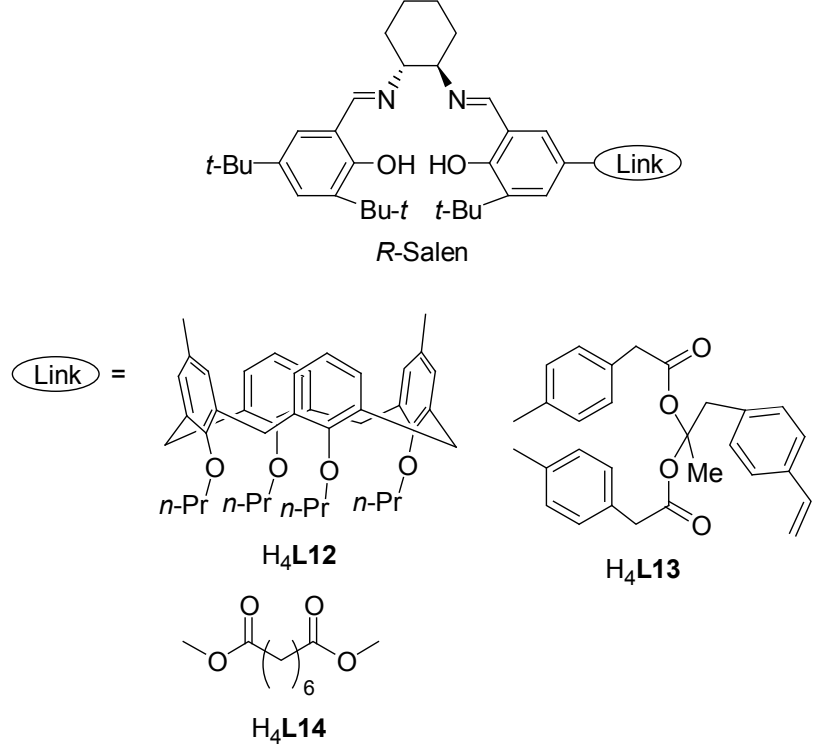

Chart 6

后，形成末端含有 Salen-Co(III)催化中心且间距可控的 栅栏式双功能催化剂，相邻两个金属核之间通过双核协 同催化作用，可以 $0.01 \mathrm{~mol} \%$ 的超低负载量催化大部分 环氧化合物的 HKR 反应, $e e$ 值基本上在 $99 \%$ 以上. 后来 Jones 等 ${ }^{[50]}$ 以上述合成方法设计了非对称的交叉型苯乙 烯-4-双 Salen 单元配体 $\mathrm{H}_{4} \mathbf{L 1 3}$, 其配合物 $\left[\mathrm{Co}_{2}^{\mathrm{III}}(\mathbf{L 3})-\right.$ $(\mathrm{OAc})_{2}$ ] 催化环氧的 HKR 反应, ee 值也在 $99 \%$ 以上(表 2 , Entry 2). $\left[\mathrm{Co}_{2}^{\text {III }}(\mathbf{L 3})(\mathrm{OAc})_{2}\right]$ 还可进一步与苯乙烯低共聚 成均相催化剂, 或与二乙烯基苯(DVB)以 $1 ： 10$ 负载生 成金属化树脂非均相催化剂, 催化的选择性与活性都未 衰减.

Kureshy 合成的长链醚环 $\mathrm{H}_{4} \mathbf{L 5} \mathbf{a}^{[6]}$ 与 $\mathrm{Co}(\mathrm{acac})_{2}$ 以及 二茂铁六氟磷酸共同作用, 得到可回收的 $\left[\mathrm{Co}_{2}^{\mathrm{III}}(\mathbf{L 5 a})-\right.$ $\left.\left(\mathrm{PF}_{6}\right)_{2}\right]$. 催化环氧的 HKR 反应，对映选择性高于相应 的 OAc 或 OTs 基配合物(表 2, Entries 3 6)，反应投料

表 2 端环氧的动力学拆分开环

Table 2 HKR of various terminal epoxides

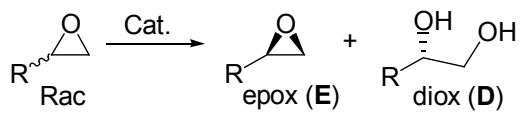

\begin{tabular}{cllcccc}
\hline Entry & \multicolumn{1}{c}{ Cat. } & Loading/mol\% & Time/h & Conv. or yield ${ }^{b} / \%(\mathbf{E}, \mathbf{D})$ & $e e^{c} / \% \mathbf{E}(\mathbf{D})$ \\
\hline 1 & $\mathrm{CH}_{2} \mathrm{Cl}$ & {$\left[\mathrm{Co}_{2}^{\text {III }}(\mathbf{L 1 2})(\mathrm{OTs})_{2}\right]$} & 0.3 & 8 & 50 & $83(83)$ \\
2 & $\mathrm{CH}_{2} \mathrm{Cl}$ & {$\left[\mathrm{Co}_{2}^{\text {III }}(\mathbf{L 1 3})(\mathrm{OAc})_{2}\right]$} & 0.02 & 7 & 51 & $-(99)$ \\
3 & $\mathrm{CH}_{2} \mathrm{Cl}$ & {$\left[\mathrm{Co}_{2}^{\text {III }}(\mathbf{L 5 a})\left(\mathrm{PF}_{6}\right)_{2}\right]$} & 0.008 & 6 & $(44,51)$ & $>99(96)$ \\
4 & $\mathrm{Ph}$ & {$\left[\mathrm{Co}_{2}^{\text {III }}(\mathbf{L 5 a})(\mathrm{OAc})_{2}\right]$} & 0.075 & 24 & $(46,41)$ & $91(90)$ \\
5 & $\mathrm{Ph}$ & {$\left[\mathrm{Co}_{2}^{\text {III }}(\mathbf{L 5 a})(\mathrm{OTs})_{2}\right]$} & 0.075 & 18 & $(46,48)$ & $94(92)$ \\
6 & $\mathrm{Ph}$ & {$\left[\mathrm{Co}_{2}^{\text {III }}(\mathbf{L 5 a})\left(\mathrm{PF}_{6}\right)_{2}\right]$} & 0.075 & 16 & $(47,48)$ & $99(92)$ \\
7 & $n-\mathrm{Bu}$ & {$\left[\mathrm{Co}_{2}^{\text {IIII }}(\mathbf{L 1 5 a})(\mathrm{OTs})_{2}\right]$} & 0.2 & 21 & 50 & $80(89)$ \\
8 & $n-\mathrm{Bu}$ & {$\left[\mathrm{Co}_{2}^{\text {III }}(\mathbf{L 1 5 b})(\mathrm{OTs})_{2}\right]$} & 0.1 & 54 & 29 & $25(43)$ \\
9 & $\mathrm{CH}_{2} \mathrm{Cl}$ & {$\left[\mathrm{Co}_{2}^{\text {III }}(\mathbf{L 1 6})_{2}\left(\mu-\mathrm{GeCl}_{3}\right)\right]$} & 0.2 & 3 & $(-, 45)$ & $>99(>85)$ \\
\hline
\end{tabular}

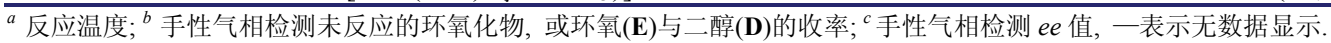


放大至克数级时催化效能未降低 ${ }^{[51]}$. Kleij 等 ${ }^{[52]}$ 通过烯烃 置换的方法设计合成了多个 Salen 多核配合物, 其中连 接型 $\left[\mathrm{Co}_{2}^{\text {III }}(\mathbf{L 5 a})(\mathrm{OTs})_{2}\right]$ 在无有机溶剂下 HKR 催化性能 好, 而大环 $\left[\mathrm{Co}_{2}^{\mathrm{III}}(\mathbf{L 5 b})(\mathrm{OTs})_{2}\right]$ 因疏水作用需在 $\mathrm{MeCN} /$ 水混合溶剂中实现 HKR 反应(Entries 7,8). 以甲醇为亲 核试剂, 两者催化环己烷氧化物的开环收率分别可达 $92 \%$ 和 $89 \%$, 但 ee 值仅为 $16 \%$ 和 $17 \%$ (Eq. 5) ${ }^{[53]}$.

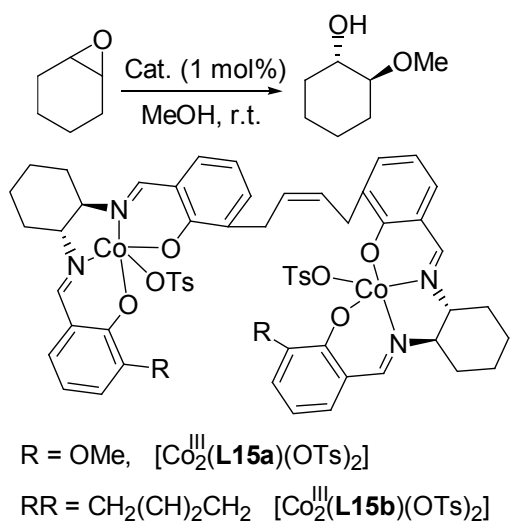

均相 Lewis 酸桥 $\left[\mathrm{Co}_{2}^{\mathrm{II}}(\mathbf{L} 16)_{2}\left(\mu-\mathrm{MX}_{3}\right)\right] \quad(\mathrm{M}=\mathrm{Al}, \mathrm{Ge}$, In) 兼具动力学拆分环氧开环和关环催化功能 ${ }^{[5]}$ (表 2 , Entry 9), 利用这一性质可以非常简便地制得对映选择 性高达 $98 \%$ 的 $(S)$-缩水甘油丁酸酯. 在催化过程中, $\mathrm{Co}(\mathrm{II})$ 核活化环氧化物, 而 Lewis 金属活化水、 $\mathrm{HCl}$ 或羧 酸亲核试剂. 与 MCM-41 分子篮共价负载后仅需延长反 应时间, 效能不受影响, 是有望工业化的非均相催化剂 (Scheme 4).

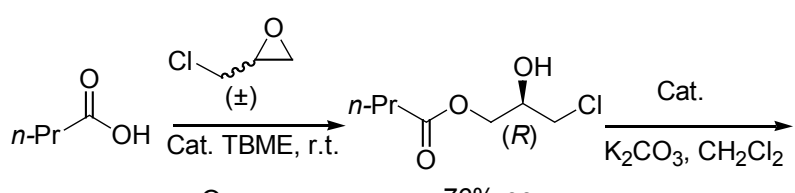<smiles>CCCC(=O)OCC1CO1</smiles>
$76 \%$ ee

(S)-glycidyl butyrate

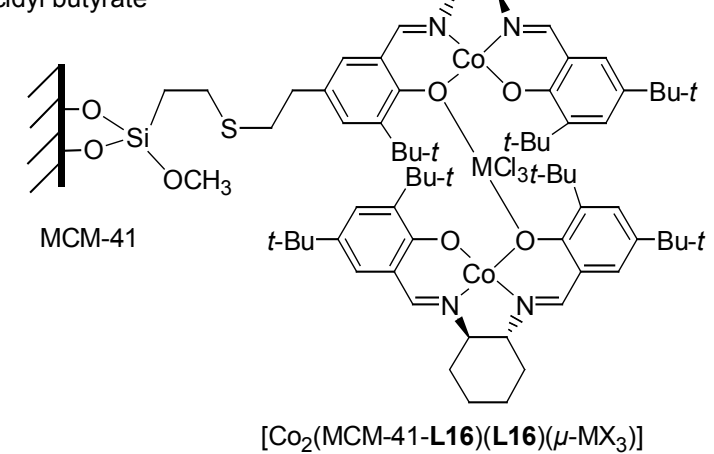

Scheme 4

除此之外, $\left[\mathrm{Co}_{2}^{\mathrm{II}}(\mathbf{L 1 6})_{2}\left(\mu-\mathrm{MX}_{3}\right)\right](\mathrm{M}=\mathrm{Al}, \mathrm{Ge}, \mathrm{In})$ 还可以担当环氧化合物与 $\mathrm{CO}_{2}$ 动力学拆分环化加成催
化剂(表 3, Entries 1 4), 但与其单核配合物[Co $\left.{ }^{\mathrm{II}}(\mathbf{L 1 6})\right]$ 相比, 催化性能没有明显差异(表 3, Entries 1，2), 适当 降低催化剂负载, 对映选择性增大但收率降低(表 3, Entry 3). 催化过程中仅涉及单个钴核对环氧进行活化, 体系中加入无机碱助催化剂, “ $\mathrm{OH} ”$ 吸收 $\mathrm{CO}_{2}$ 形成 $\mathrm{HCO}_{3}^{-}$，再对环氧进行亲核进攻，催化效能稍有提高 (Entry 4) ${ }^{[54]}$.

Jing 等 ${ }^{[55]}$ 将联萗 Salen 新型大环配合物 $\left[\mathrm{Co}_{2}^{\mathrm{III}}(\mathbf{L} \mathbf{1 7})-\right.$ $(\mathrm{OAc})_{2}$ （Chart 7)用于催化 $\mathrm{CO}_{2}$ 与环氧的环化加成, BINOL 和 Salen 配体之间可能也存在相互促进作用, 手性 构型一致时对映选择性稍高能达到 54\%(表 3, Entry 5).

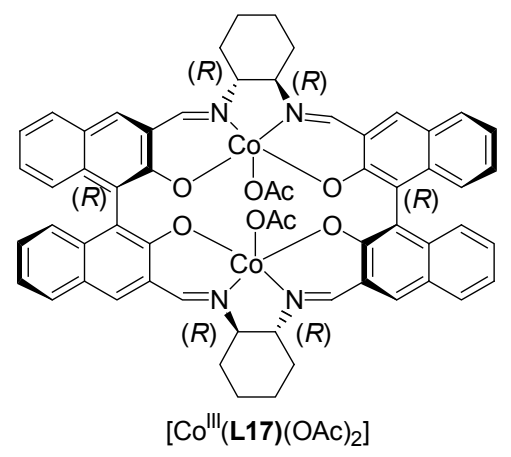

Chart 7

表 $3 \mathrm{CO}_{2}$ 与环氧丙烷的动力学拆分环化加成

Table 3 HKR asymmetric cycloaddition of $\mathrm{CO}_{2}$ and propylene oxide

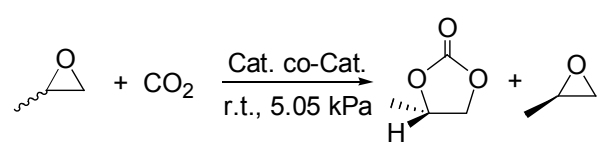

\begin{tabular}{|c|c|c|c|c|}
\hline Entry & Cat. & co-Cat. & $\begin{array}{l}\text { Loading } / \mathrm{mol} \% \\
\text { (co-Cat.) }\end{array}$ & $\begin{array}{l}\text { Yield } \\
(e e) / \%\end{array}$ \\
\hline 1 & {$\left[\mathrm{Co}_{2}^{\mathrm{II}}(\mathbf{L 1 6})_{2}\left(\mu-\mathrm{MX}_{3}\right)\right]$} & 一 & $0.5(0)$ & $19(68)$ \\
\hline 2 & {$\left[\mathrm{Co}^{\mathrm{II}}(\mathbf{L} \mathbf{1 6})\right]$} & - & $0.5(0)$ & $20(73)$ \\
\hline 3 & {$\left[\mathrm{Co}^{\mathrm{II}}(\mathbf{L} 16)\right]$} & - & $0.1(0)$ & $11(81)$ \\
\hline 4 & {$\left[\mathrm{Co}^{\mathrm{II}}(\mathbf{L} 16)\right]$} & {$[\mathrm{BMIm}] \mathrm{OH}^{a}$} & $0.1(0.01)$ & $45(83)$ \\
\hline 5 & {$\left[\mathrm{Co}_{2}^{\mathrm{III}}(\mathbf{L 1 7})(\mathrm{OAc})_{2}\right]$} & $\mathrm{PTAT}^{b}$ & $0.05(0.1)$ & $43(54)$ \\
\hline
\end{tabular}

锌核面面相对, 含 $C_{3}$-对称轴的 $\left[\mathrm{Zn}_{2}(\mathbf{L 1 8})_{2}(\mu-\mathrm{OTf})_{3}\right] \cdot$ OTf 催化氨酰苯酯的动力学拆分酯交换反应(Scheme $5)^{[56]}$, 原位催化时, 当 $\mathrm{Zn}(\mathrm{OTf})_{2}$ 盐分别被 $\mathrm{Zn}(\mathrm{OAc})_{2}$ 和 $\mathrm{Zn}\left(\mathrm{CF}_{3} \mathrm{COO}\right)_{2}$ 替代时, 反应的选择因子依次递增 $(2.0$, $4.5,5.1)$. 这类 $C_{3}$-对称轴配体与 $\mathrm{Cu}(\mathrm{II})$ 形成高对映选择 性的 Lewis 催化剂 ${ }^{[57]}$.

传统的 $\mathrm{C}-\mathrm{F}$ 键的形成需要苛刻的反应条件，而环 氧的动力学拆分氟开环可以在较温和的条件下制备手 性纯的 $\beta$-氟醇. Doyle 等 ${ }^{[58]}$ 以六氟异丙醇(HFIP)作氟源, 以 1,5 -二氮杂双环 $[4,3,0]$ 壬-5-烯(DBN)为助催化剂, 催 


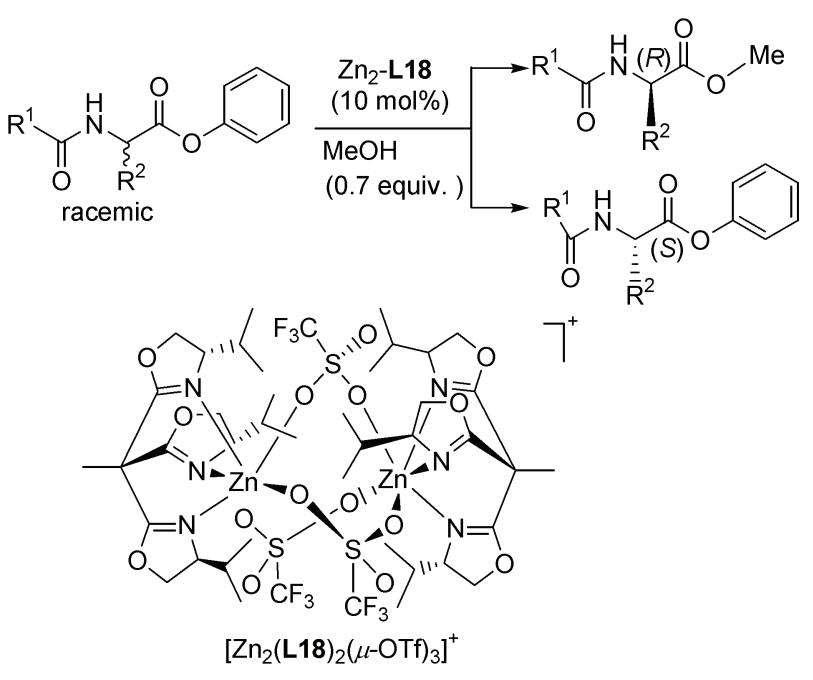

Scheme 5

化环氧的对映选择性氟开环反应, 单钴催化剂呈现一个 正的非线性效应. 后来该课题组 ${ }^{[59]}$ 又设计了具有协同 作用的 $\left[\mathrm{Co}_{2}{ }_{2}\right.$ (L14) $]$, 对映选择性和活性都较以前的单 核配催化剂提高了很多, 反应时间也缩短了 $95 \%$ 以上. Doyle 首次提出了非活化 Salen-Co 主要以氟桥二聚状态 存在, 手性钴核与同-或异一手性环氧的结合速率相等, 但只有后者才可与 DBN-Co-FHF 活性亲核结构反应, 亲 核进攻开环过程为速率控制步骤(Scheme 6). 此种方式 合成活体细胞用 PET 放射性示踪剂 F-MISO 最高可达 $40 \%$ 的收率和 $93 \%$ 的 $e e$ 值.
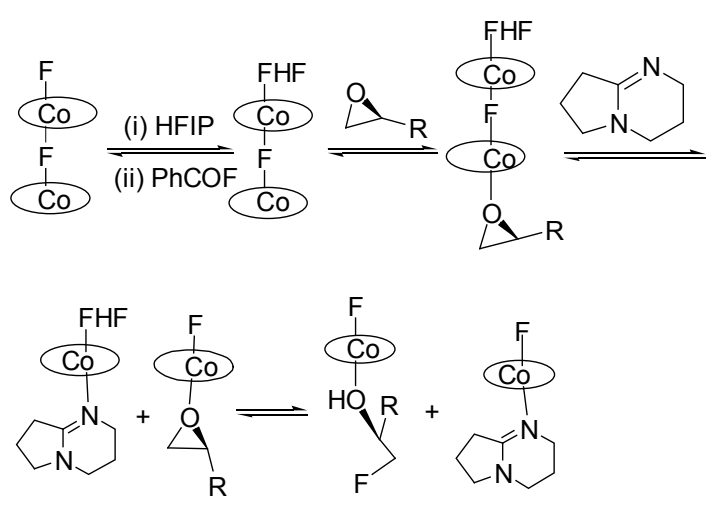

Scheme 6

\section{3 氧化型催化剂}

除了以上通过对消旋环氧化合物的动力学拆分水 解反应外, 烯烃的对映选择性氧化也是获得手性环氧化 合物的可行方法. 在众多已报道的催化剂中, 有机锰Salen 配合物是目前被认为这类反应中较好的催化剂 ${ }^{[60]}$.

双 Salen-Mn 催化剂(Chart 8) 在这类反应中的应用 受到了广泛关注. 2004 年, Tang 等 ${ }^{[61]}$ 先将 $(R, R)$-手性二 胺的其中一个氨基与醛缩合, 再与带有连接单元的双二
醛进行缩合，合成的双核锰配合物 $\mathrm{Mn}_{2}^{\text {III }} \mathrm{Cl}_{2} \mathbf{L 1 9 a} \sim$ $\mathrm{Mn}_{2}^{\mathrm{III}} \mathrm{Cl}_{2} \mathbf{L 1 9 c}$ ), 用于催化反式均二苯乙烯的环氧化反 应(表 4, Entries 1 3). 手性苯二胺 Salen 单元配合物 $\left[\mathrm{Mn}_{2}^{\text {III }} \mathrm{Cl}_{2}(\mathbf{L 1 9 a})\right]$ 的对映选择性高于环己烷二胺 $\left[\mathrm{Mn}_{2}^{\mathrm{III}} \mathrm{Cl}_{2}(\mathbf{L 1 9 b})\right]$, 当亚胺基上的位阻增大时, 对映选 择性升高但伴随着收率降低(表 4, Entry 3).

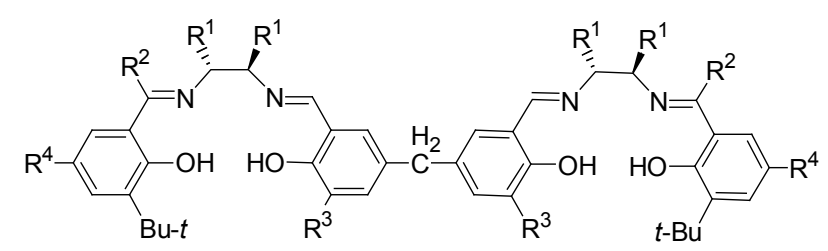

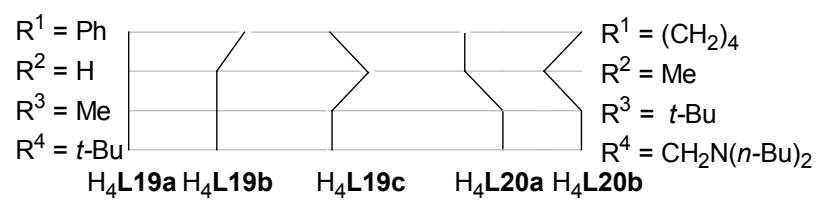

Chart 3

该小组在末端连接叔胺基, 合成了改良的且有相转 移能力的 $\left.\left[\mathrm{Mn}_{2}^{\mathrm{III}} \mathrm{Cl}_{2} \mathbf{L 2 0 a}, \mathrm{Mn}_{2}^{\mathrm{III}} \mathrm{Cl}_{2} \mathbf{L 2 0 b}\right)\right]$, 催化对映选 择性和活性较以前的 $\mathrm{Mn}_{2}^{\text {III }} \mathrm{Cl}_{2} \mathbf{L 1 9 a} \sim \mathrm{Mn}_{2}^{\text {III }} \mathrm{Cl}_{2} \mathbf{L 1 9 c}$ 大 大提高了(表 4, Entries 5 7), 离子液体是比 PyNO 更好 的共催化剂(表 4, Entries 6,7). 对于取代的色烯，催化 活性和 $e e$ 值都较理想(表 4, Entries 5 7), 六次回收循环 使用催化效能未衰竭 ${ }^{[62]}$. Trivedi 等 ${ }^{[63]}$ 将联吡啶桥(Bpy) 或菲罗啉 (Phen) 桥 $\left[\mathrm{Mn}_{2}^{\mathrm{III}}((R)-\mathrm{BINOL})_{3}\left(\mu-1,1^{\prime}-\mathrm{Bpy} /\right.\right.$ Phen $)_{2}$ ] 较不理想的催化性能归各于催化过程中可能出 现的离子或自由基中间体.

含 $C_{2}$ 对称轴的手性 $\left[\mathrm{Mn}^{\mathrm{III}} \mathrm{Mn}^{\mathrm{IV}}((R)-\mathbf{L 2 1})_{2}(\mu-\mathrm{O})_{2}\right]-$ $\left(\mathrm{PF}_{6}\right)_{3}{ }^{[64]}$ 是广谱的 $\mathrm{H}_{2} \mathrm{O}_{2}$ 氧化催化剂, 可用来催化烯烃、 烷烃以及醇的氧化, 但收率和对映选择性都不高(表 4, Entries 8,9). Kwong 等 ${ }^{[65]}$ 合成了两端含有 $(R)$-派烯 $[5,6]$ 吡啶的手性多吡啶配体 L22 (Chart 9)，与铁配合生成缠 绕型 $\mathrm{Fe}(\mathrm{III})$ 氧桥铁催化剂 $\left[\mathrm{Fe}_{2} \mathrm{O}(\mathbf{L 2 2}) \mathrm{Cl}_{4}\right]$, 催化 $\mathrm{H}_{2} \mathrm{O}_{2}$ 对 环氧的对映选择性氧化, 活性和化学选择性较高(表 4, Entry 10).

Kwong 等 ${ }^{[66]}$ 对联吡定派烯 L23d 进行修饰，设计了 中间带刚性苯基的 L23a L23c (Chart 10), 合成了对空 气不敏感的 $\left[\mathrm{Mn}_{2}^{\mathrm{II}}(\mathbf{L 2 3 a} \sim \mathbf{L 2 3 c})_{2}\left(\mathrm{H}_{2} \mathrm{O}\right)_{4}\right]\left(\mathrm{ClO}_{4}\right)_{4}$ 螺旋, 催化烯烃的环氧化可达 $77 \% \sim 81 \%$ 收率，对于位阻较小 的烯烃甚至可达 95\%。但基本上没有对映选择性. Rodríguez 等 ${ }^{[67]}$ 通过 $(S)$-L23d 与 $\mathrm{Mn}$ 盐直接反应合成了 $\left[\mathrm{Mn}_{2}^{\mathrm{II}} \mathrm{Cl}_{2}((S)-\mathbf{L} 23 \mathbf{d})_{2}(\mu-\mathrm{Cl})_{2}\right]$ 和 $\left[\mathrm{Mn}_{2}^{\mathrm{II}}((S)-\mathbf{L 2 3 d})_{2}(\mu-\right.$ $\left.\mathrm{OAc})_{3}\right]\left(\mathrm{PF}_{6}\right)$, 前者催化活性较高, 但化学选择性和对映 选择性不理想. 后者的化学选择性稍高而活性很低(表 4, Entries 11, 12). 
表 4 烯烃的环氧化

Table 4 Asymmetric of various alkenes

\begin{tabular}{|c|c|c|c|c|c|c|c|}
\hline Entry & Substrate & Cat. & Oxidate & Co-Cat. & Loading $/ \mathrm{mol} \%$ & Conf. & Yield $(e e) / \%$ \\
\hline 1 & $\mathrm{Ph}-$ & {$\left[\mathrm{Mn}_{2}^{\text {III }} \mathrm{Cl}_{2}(\mathbf{L 1 9 a})\right]$} & $\mathrm{NaClO}$ & $\mathrm{PyNO}^{a}$ & 2.5 & $(1 R, 2 R)$ & $85(5)$ \\
\hline 2 & $\mathrm{Ph} \leadsto$ & {$\left[\mathrm{Mn}_{2}^{\mathrm{III}} \mathrm{Cl}_{2}(\mathbf{L 1 9 b})\right]$} & $\mathrm{NaClO}$ & PyNO & 2.5 & $(1 R, 2 R)$ & $67(10)$ \\
\hline 3 & $\mathrm{Ph}^{-}$ & {$\left[\mathrm{Mn}_{2}^{\mathrm{III}} \mathrm{Cl}_{2}(\mathbf{L 1 9 c})\right]$} & $\mathrm{NaClO}$ & PyNO & 2.5 & $(1 R, 2 R)$ & $43(40)$ \\
\hline 4 & $\mathrm{Ph}-$ & {$\left[\mathrm{Mn}_{2}^{\mathrm{III}} \mathrm{Cl}_{2}(\mathbf{L 2 0 a})\right]$} & $\mathrm{NaClO}$ & PyNO & 5 & $(1 R, 2 R)$ & $84(21)$ \\
\hline 5 & & {$\left[\mathrm{Mn}_{2}^{\mathrm{III}} \mathrm{Cl}_{2}(\mathbf{L 2 0 a})\right]$} & $\mathrm{NaClO}$ & PyNO & 5 & $(3 R, 4 R)$ & $89(93)$ \\
\hline 6 & & {$\left[\mathrm{Mn}_{2}^{\mathrm{III}} \mathrm{Cl}_{2}(\mathbf{L 2 0 a})\right]$} & $\mathrm{NaClO}$ & {$\left[\mathrm{BMIM} \mathrm{PF}_{6}\right.$} & 5 & $(3 R, 4 R)$ & $93(94)$ \\
\hline 7 & $\mathrm{Cl}^{-}$ & {$\left[\mathrm{Mn}_{2}^{\mathrm{III}} \mathrm{Cl}_{2}(\mathbf{L 2 0 b})\right]$} & $\mathrm{NaClO}$ & {$[\mathrm{BMIM}] \mathrm{PF}_{6}$} & 5 & $(3 R, 4 R)$ & $93(94)$ \\
\hline 8 & Indene & {$\left[\mathrm{Mn}^{\mathrm{III}} \mathrm{Mn}^{\mathrm{IV}}((R)-\mathbf{L 2 1})_{2}(\mu-\mathrm{O})_{2}\right] \cdot\left(\mathrm{PF}_{6}\right)_{3}$} & $\mathrm{H}_{2} \mathrm{O}_{2}$ & - & 1.5 & $(1 R, 2 S)$ & $4(17)$ \\
\hline 9 & Indene & {$\left[\mathrm{Mn}^{\mathrm{III}} \mathrm{Mn}^{\mathrm{IV}}((R)-\mathbf{L} 21)_{2}(\mu-\mathrm{O})_{2}\right] \cdot\left(\mathrm{PF}_{6}\right)_{3}$} & $\mathrm{H}_{2} \mathrm{O}_{2}$ & 草酸钠 & 1.5 & $(1 R, 2 S)$ & $16(4)$ \\
\hline 10 & $\mathrm{PhCH}=\mathrm{CH}_{2}$ & {$\left[\mathrm{Fe}_{2} \mathrm{O}(\mathbf{L 2 2}) \mathrm{Cl}_{4}\right]$} & $\mathrm{H}_{2} \mathrm{O}_{2}$ & - & 2 & $(1 R, 2 R)$ & $95(43)$ \\
\hline 11 & $\mathrm{PhCH}=\mathrm{CH}_{2}$ & {$\left[\mathrm{Mn}_{2}^{\mathrm{II}} \mathrm{Cl}_{2}((S)-\mathbf{L} \mathbf{2 3 d})_{2}(\mu-\mathrm{Cl})_{2}\right]$} & $\mathrm{PAA}^{b}$ & - & 1 & $(1 S, 2 S)$ & $65(10)$ \\
\hline 12 & $\mathrm{PhCH}=\mathrm{CH}_{2}$ & {$\left[\mathrm{Mn}_{2}^{\mathrm{II}}((S)-\mathbf{L} 23 d)_{2}(\mu-\mathrm{OAc})_{3}\right]\left(\mathrm{PF}_{6}\right)$} & PAA & - & 1 & $(1 S, 2 S)$ & $10(19)$ \\
\hline
\end{tabular}

${ }^{a} \mathrm{PyNO}=$ 吡啶氮氧化物; ${ }^{b} \mathrm{PAA}=$ 过氧乙酸.

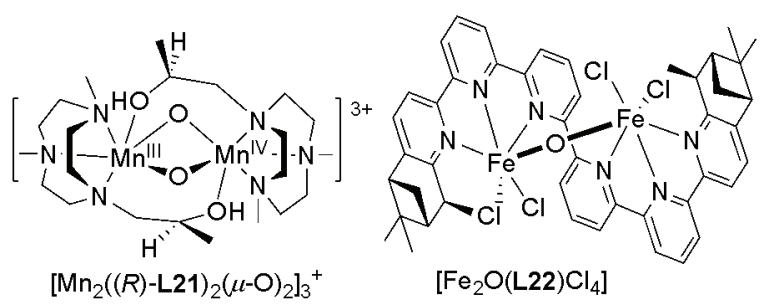

Chart 9

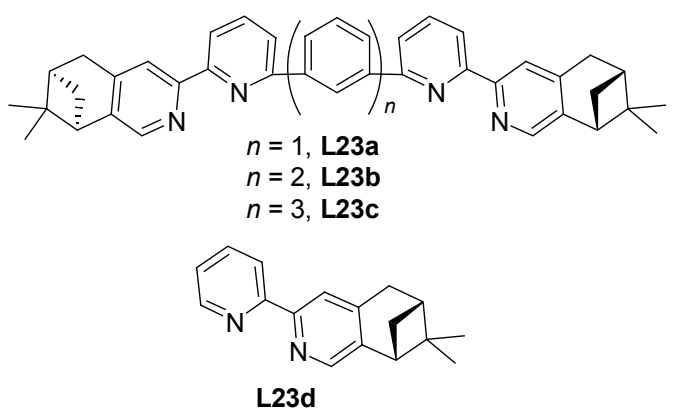

Chart 10

Legros 等 ${ }^{[68]}$ 在对映选择性硫氧化的实现过程中, 使 用了通过原位合成的铁催化剂(Eq. 6). 线性相关实验结 果为正的非线性效应. 该作者认为, 起催化作用的可能

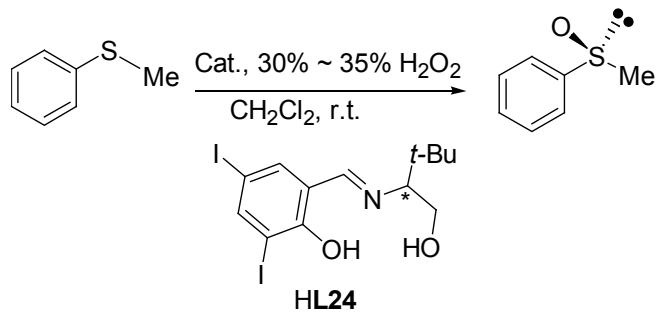

是类似于已经报道的 $\left[\mathrm{Fe}_{2}(\mathbf{L} \mathbf{2 4})(\mu-\mathrm{O})(\mu-\mathrm{OTs})\right]$ 手性双核铁 催化剂 ${ }^{[69]}$.

$\left[\left(\mathrm{V}^{\mathrm{V}} \mathrm{O}\right)_{2}\left(\left(R_{a}, S, S\right)-\mathbf{L} \mathbf{2 5} \mathbf{a}, \mathbf{b}\right)(\mathrm{OH})_{2}\right]$ 催化 2-萗酚氧化偶 合生成(S)-BINOL (Chart 11), $\left(R_{a}, S, S\right)$-L25a 的配合物催 化收率几乎为定量, 高于相应的 $\left(R_{a}, S, S\right)$-L25b $(56 \%$ 收 率), 但对映选择性 $(90 \% e e)$ 不及 $\left(R_{a}, S, S\right)-\mathbf{L} 25 \mathbf{b}(97 \% e e)$. 对于 6-苯基-2-萗酚底物, 两者催化的收率都为定量, ee 值也分别为 $86 \%$ 和 $93 \%{ }^{[70]}$.

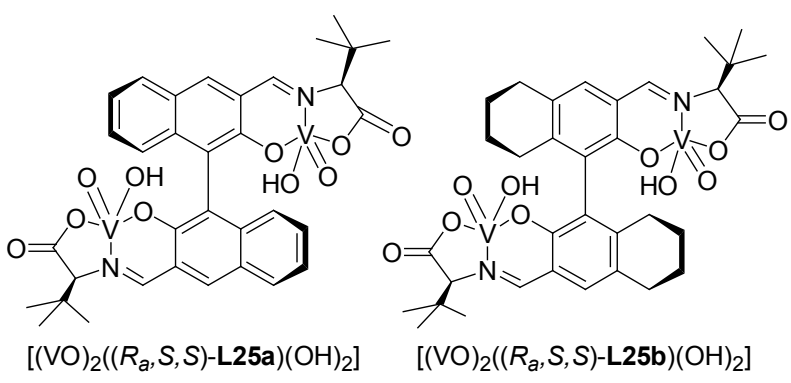

Chart 11

$\left[\left(\mathrm{V}^{\mathrm{V}} \mathrm{O}\right)_{2}\left(\left(R_{\boldsymbol{a}}, S, S\right.\right.\right.$-L 25a $\left.)(\mathrm{OH})_{2}\right]$ 还可作为 Lewis 酸催化 剂 ${ }^{[71]}$, 氮气氛围下催化 $\beta$-荟酚或弜哚与亚胺的 FriedelCrafts 反应，最高可达 $91 \% e e$ 值. 酯基上的 $\mathrm{C}=\mathrm{O}$ 双键、 $\mathrm{V}$ 上的 “ $\mathrm{OH}$ ” 配体及联菜的大 $\pi$ 键结构改变(即 $\left[\left(\mathrm{V}^{\mathrm{V}} \mathrm{O}\right)_{2}\left(\left(R_{\boldsymbol{a}}, S, S-\mathbf{L} 25 \mathbf{b}\right)(\mathrm{OH})_{2}\right]\right)$ 后，催化选择性都有所下 降. 该 Friedel-Crafts 反应基本上没有偶联副产物, 但对 于 $N$-烷基吲哚底物的催化反应没有对映选择性. 反应 通过一个双活化机理(Chart 12), N-V 共价键的形成对 于对映选择性非常重要. 


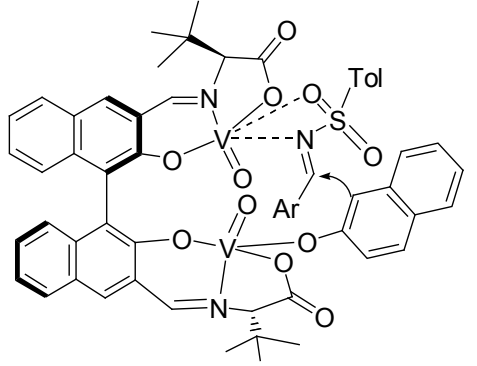

Chart 12

除了以上对映选择性合成反应以外, 手性双核氧化 催化剂能参与对底物的动力学拆分氧化. 含八氮齿及三 个 $S$-手性碳的配体 L26 (Chart 13) 柔韧的构型使其具有较 好的儿茶酚催化活性. $\left[\mathrm{Cu}_{2}(\mathbf{L 2 6})\right]\left(\mathrm{ClO}_{4}\right)_{4}$ 对 $L$-和 $D$-构型的 儿茶酚及其相应的酯衍生物底物的氧化速率常数存在差 异. 一般氧化 $L$-型的速率快, 对于 $L$-多巴(Dopa)这种选 择性可达 33\%, 但比其三核配合物(75\%)选择性低 ${ }^{[72]}$.

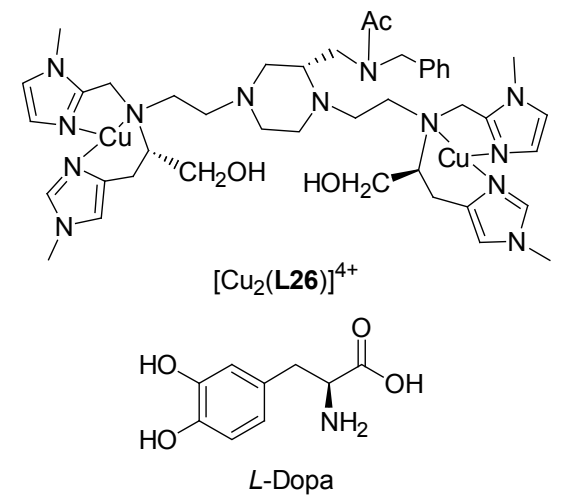

Chart 13

\section{4 不对称聚合反应催化剂}

由金属酚或烷氧配合物催化的环酯开环聚合, 是重 要的控制性聚合策略. 耐热且高机械性能的手性高分子 的立体控制性制备，仍然是当前一大挑战. 呈伞状配位 的手性配合物 $\left[\mathrm{Zn}_{2}(\mathbf{L} 27)(\mu-\mathrm{Cl})(\mathrm{Cl})_{2}\right]$ (Chart 14)对 $\varepsilon$-己内 酮的开环聚合催化, 并没表现出对映选择性, 而且也只 有中等的催化活性， $100{ }^{\circ} \mathrm{C}$ 下反应 $115 \mathrm{~h}$ 后仅得 $55 \%$ 的 转化率 ${ }^{[73]}$.

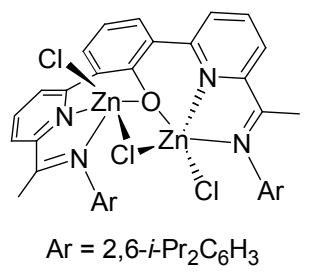

$\left[\mathrm{Zn}_{2}(\mathbf{L 2 7})(\mu-\mathrm{Cl})(\mathrm{Cl})_{2}\right]$

Chart 14
Nozaki 等 ${ }^{[74]}$ 合成了与 Trost 催化剂 $\left[\mathrm{Zn}_{2}(\mathbf{L 3})(\mathrm{Et})\right]$ 结 构相似仅在对称性上有所差异的 $\left[\mathrm{Zn}_{2}((S)-\mathbf{L 2 8})_{2}(\mathrm{Et})_{2}\right]$, 其催化环己烷环氧化物与 $\mathrm{CO}_{2}$ 的共聚, 活性和对映选择 性不及 $\left[\mathrm{Zn}_{2}((S)-\mathbf{L 2 8})(\mathrm{Et})(\mathrm{OEt})\right]$ (Eq. 7).

手性配体 HL28 在 Eq. 7 反应中也存在手性放大现 象 ${ }^{[75]}$, 这也是首个被发现的主链不对称聚合反应中的 非线性效应. 当采用内消旋或外消旋双核作为催化剂 时，对映选择性和活性都大大下降. 于是作者认为，同 手性双核配物分解后再重组, 形成更稳定但催化活性低 的异手性双核 $\left[\mathrm{Zn}_{2}((R)-\mathbf{L} 28)((S)-\mathbf{L} 28)\right]$.

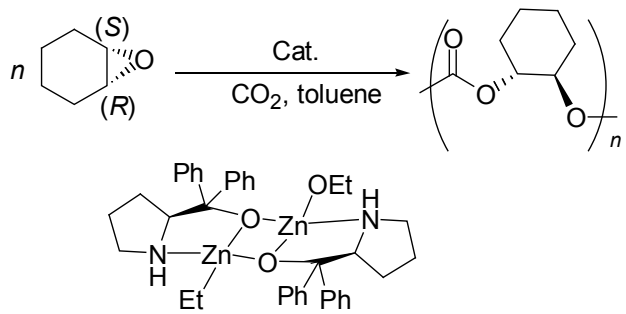

$\left[\mathrm{Zn}_{2}((S)-\mathrm{L} 28)_{2}(\mathrm{Et})(\mathrm{OEt})\right]$

Nozaki 等 ${ }^{[76]}$ 尝试采用与 $\mathrm{H}_{4} \mathbf{L 1 4}$ 相似但中间亚甲基 的长度有差异的双 Salen-Co(III), 催化环氧化合物与 $\mathrm{CO}_{2}$ 的区域选择性聚合, 并与鎓盐辅助的单核 Salen 配 合物 ${ }^{[77]}$ 的催化作了简单比较. 两端的 Salen 构型不一致 时，催化活性较高，两个 Salen 单元空间距离中间含 4 个亚甲基长度时，区域选择性好，使用距离 10 个亚甲基 长度的催化剂时聚合物主链规整性较好. 作者用双金属 活化机理解释了上述催化过程(Scheme 7). 而在鎓盐 $[\mathrm{PPN}] \mathrm{Cl}$ 的助催化作用下，单核或双核 Salen 配合物都趋 向于单金属催化机理途径 ${ }^{[78]}$.

虽然环氧的 HKR 开环反应催化研究已经非常成熟, 但高对映选择性的拆分聚合催化剂近几年才发展起来. Coates 等基于环氧水解双活化机理发展了 $\left[\mathrm{Co}_{2}^{\text {III }}(\mathbf{L 2 9}) \mathrm{Cl}_{2}\right]$ (Chart 15) 催化剂, 联萗轴的可旋转性 使得两钴核之间的空间具有可适性. $\left[\mathrm{Co}_{2}^{\mathrm{III}}(\mathbf{L 2 9}) \mathrm{Cl}_{2}\right]$ 催 化环氧动力学拆分聚合, 对大多数烷基、芳基或烯基离 环氧较远的环氧底物，反应完成时间基本上在 $0.5 \mathrm{~h}$, 同 系指数 $(\mathrm{mm})$ 高达 $98.8 \%$, 聚合物 $e e$ 值基本上都在 $98 \%$ 以 上(表 5, Entries 1,2,4,5), 而对于 1-环丙氧基乙烯, $e e$ 和 $m m$ 值都不高(表 5, Entry 3) ${ }^{[79]}$. Rac- $\left[\mathrm{CO}_{2}^{\mathrm{III}} \mathrm{Cl}_{2}(\mathbf{L 2 9})\right]$ 则是 全同异构催化剂，催化反应定量进行，生成可控分子量 热稳定的全同异构聚合物(表 5, Entries $7 \sim 10)^{[80]}$. 对于 多取代或非端环氧的底物来说，这类催化剂没有催化活 性.

Otero 课题组 ${ }^{[81]}$ 合成了以 $\left[\mathrm{Zn}_{2}((R)-\mathbf{L 3 0})_{2}\left(\mathrm{CH}_{2} \mathrm{SiMe}_{2}\right)_{2}\right]$ 为代表的非甲基锌与手性二咪唑基醇蝎形二聚配合物, 


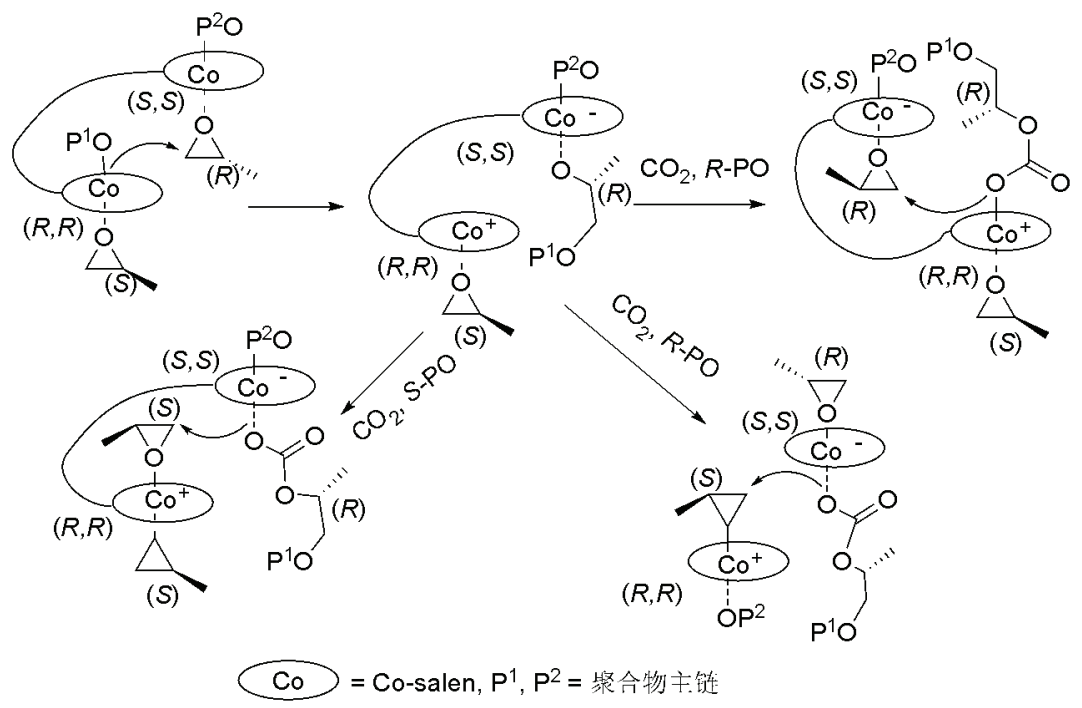

Scheme 7

表 5 环氧的 HKR 或异构聚合

Table 5 HKR or isoselective polymerization of terminal epoxides

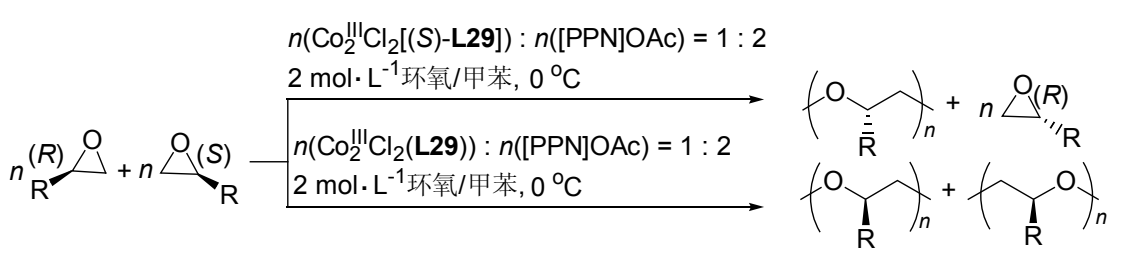

\begin{tabular}{clccccc}
\hline Entry $^{a}$ & \multicolumn{1}{c}{$\mathrm{R}$} & Loading $/ \mathrm{mol} \%$ & Time $/ \mathrm{min}$ & Conv./\% & $e e / \%$ & $\mathrm{~mm} / \%$ \\
\hline 1 & $\mathrm{Me}$ & 0.025 & 15 & 34 & 99 & 98 \\
2 & $\mathrm{Et}$ & 0.10 & 14 & 22 & 99 & 99 \\
3 & Vinyl & 0.10 & 32 & 45 & 81 & 75 \\
4 & $4-$ Butenyl & 0.15 & 11 & 35 & 99 & 99 \\
5 & $\mathrm{Ph}$ & 0.10 & 30 & 34 & 96 & 94 \\
6 & $\mathrm{Me}$ & 0.10 & $3.5^{a}$ & $>99$ & - & $>99$ \\
7 & $\mathrm{Et}$ & 0.10 & $28^{a}$ & $>99$ & - & $>99$ \\
8 & Vinyl & 0.10 & $1.7^{a}$ & $>99$ & - & 85 \\
9 & $\mathrm{Ph}$ & 0.10 & & - & 93 \\
\hline
\end{tabular}

${ }^{a}$ Time are in $\mathrm{h}$.

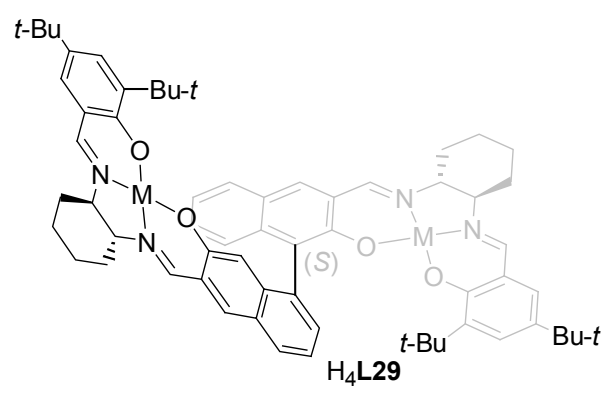

Chart 15

作为消旋丙交酯(LAC)在 THF 中的活性开环聚合引发 剂 (Eq. 8), 位阻较大的烷基锌试剂对应的基体中消旋 键机率 $\operatorname{Pr}$ 值高 $(0.69 \sim 0.77)$. 在甲苯溶剂中 $\operatorname{Pr}$ 稍低. 同 样的甲基锌容易生成催化活性较低的单核配合物.

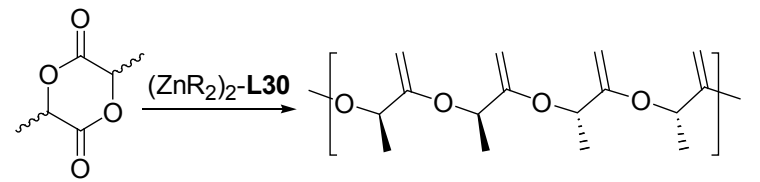




\section{5 其它催化剂}

$(1 R, 2 S)$ - 顺式-1-亚胺基苑醇型双核 $\left[\mathrm{Cu}_{2}^{\mathrm{II}}(\mathbf{L 3 1})_{2}\right]$ (Chart 16)催化苯乙烯的环丙基化活性高(表 6, Entry 1), syn : trans $\approx 1: 2$, 但对映选择性差 $(<2 \% e e)$, 较作者 同期研究的手性席夫碱单核催化剂活性没有明显的优 势, 对映选择性甚至要差很多 ${ }^{[82]}$.

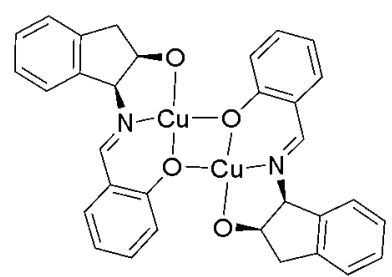

$\left[\mathrm{Cu}_{2}(\mathrm{~L} 31)_{2}\right]$

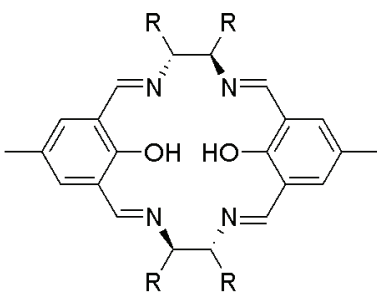

$\mathrm{RR}=\left(\mathrm{CH}_{2}\right)_{4} \mathrm{H}_{2} \mathrm{~L} 32 \mathrm{a}$

\section{Chart 16}

Gao 等 ${ }^{[83]}$ 尝试将大环 Salen-Co(II/III) 和 $\mathrm{Mn}(\mathrm{II} / \mathrm{III})$ 双 核配合物应用到烯烃的不对称环丙基化，大环平面像酶 的活性中心一样将底物分子固定, 表现出非常高的对映 选择性(表 6, Entries 2 9). Co(III)活性与对映选择性相 对而言最好, 同样苯二胺配体 $\mathrm{H}_{2} \mathbf{L} \mathbf{2}$ b 的催化效能比环 己基二胺配体 $\mathrm{H}_{2} \mathbf{L 3 2 a}$ 的高(表 6, Entries 5,9). 作者认
为，一分子金属催化剂与两个重氮基醋酸酯分子作用, 形成一对含两个相互平行金属-卡宾双键的顺反镜面异 构中间体，烯烃从平行的位置与金属-卡宾双键反应， 分别形成对映选择性较高的 trans 或 cis 式产物.

$\alpha$-偶氮酯 $\mathrm{N}-\mathrm{H}$ 不对称插入反应是合成 $\alpha$-氨基酸的 一种重要手段, $\left(S_{a}, S, S\right)$ - $\mathbf{L 3 3}$ 和亚铜盐共催化 $\mathrm{N}-\mathrm{H}$ 不对 称插入反应效能高底物适应性广 ${ }^{[84]}$. 非线性实验结果 为微小的正非线性相关, $X$ 衍射单晶结构证实了 $C_{2}$ 对称 轴手性 $\left[\mathrm{Cu}_{2}^{\mathrm{I}}\left(S_{a}, S, S-\mathbf{L} 33\right)_{2}\right]$ [对催化过程对映选择性有较 大贡献(Eq. 9).

\section{2 总结与展望}

第一过渡系金属手性双核催化剂，特别是双核间协 同作用较强的催化剂, 在许多不对称反应上有着较为成 功的应用，但目前仍还有一些问题有待解决. 首先，手 性双核催化剂的适用性. 双核催化剂并不是对所有反应 都表现出高于单核手性催化剂的催化性能，而且，本文 所归纳的反应只是不对称反应中的冰山一角，第一过渡 系金属手性双核催化剂在其它反应上的催化应用，仍有 待众多学者去开拓和研究. 其次, 一些难以反应的低活 性底物的局限性. 如环氧化合物中的环氧丁烷或环氧丁

表 6 烯烃的不对称环丙基化

Table 6 Asymmetric cyclopropanation of terminal alkene

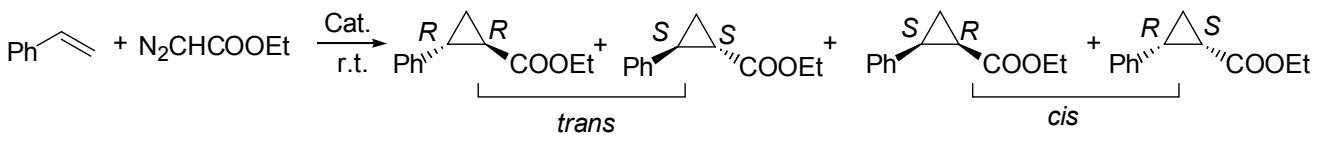

\begin{tabular}{|c|c|c|c|c|c|c|}
\hline Entry & Cat. & Loading/mol\% & Yield $/ \%$ & cis/trans ratio & $e e(c i s)^{a} / \%$ & $e e(\text { trans })^{b} / \%$ \\
\hline 1 & {$\left[\mathrm{Cu}_{2}(\mathbf{L 3 1})_{2}\right]$} & 1 & 88 & $31: 69$ & 1.46 & 0.85 \\
\hline 2 & {$\left[\mathrm{Mn}_{2}^{\mathrm{II}}-(\mathbf{L 3 2 a})\right]$} & 5 & 70 & $64: 36$ & 57 & 51 \\
\hline 3 & {$\left[\mathrm{Mn}_{2}^{\mathrm{III}}-(\mathbf{L 3 2 a})\right]$} & 5 & 62 & $68: 32$ & 58 & 56 \\
\hline 4 & {$\left[\mathrm{Co}_{2}^{I I}-(\mathbf{L 3 2 a})\right]$} & 5 & 90 & $72: 28$ & 92 & 86 \\
\hline 5 & {$\left[\mathrm{Co}_{2}^{\mathrm{III}}-(\mathbf{L 3 2} \mathbf{a})\right]$} & 5 & 92 & $74: 26$ & 94 & 88 \\
\hline 6 & {$\left[\mathrm{Mn}_{2}^{\mathrm{II}}-(\mathbf{L 3 2 b})\right]$} & 5 & 84 & $67: 33$ & 86 & 82 \\
\hline 7 & {$\left[\mathrm{Mn}_{2}^{\mathrm{III}}-(\mathbf{L} 32 \mathbf{b})\right]$} & 5 & 69 & $69: 31$ & 70 & 78 \\
\hline 8 & {$\left[\mathrm{Co}_{2}^{\mathrm{II}}-(\mathbf{L} \mathbf{3 2 b})\right]$} & 5 & 93 & $70: 30$ & 92 & 89 \\
\hline 9 & {$\left[\mathrm{Co}_{2}^{\mathrm{III}}-(\mathbf{L 3 2 b})\right]$} & 5 & 94 & $76: 24$ & 90 & 94 \\
\hline
\end{tabular}

${ }^{a}(1 S, 2 R)$ 为主要对映异构体; ${ }^{b}(1 R, 2 R)$ 为主要对映异构体.

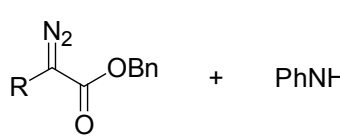

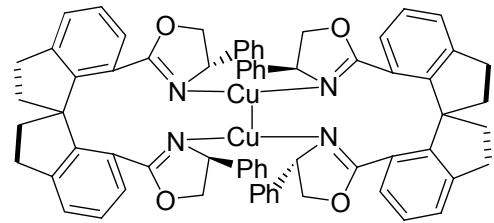

$\left[\mathrm{Cu}_{2}^{\prime}\left(S_{\mathrm{a}}, \mathrm{S}, \mathrm{S}-\mathrm{L} \text { 33 }\right)_{2}\right]$ 
烯，或烯烃中的非端烯烃. 再次，一些同时涉及化学、区 域和对映的多重选择性. 如文中的选择性氧化或卡宾反

应、多组分反应，非常难以多者兼顾.

虽然如此，第一过渡系金属手性双核催化剂的发 展，为不对称合成带来更多的机遇. 设计和合成高效稳 定可回收利用的第一过渡系金属手性双核催化剂是降 低手性合成的成本、减少重金属污染、提高手性选择性 和催化效能的有效途径, 是这类配合物的生理医学应用 前提. 基于自然界普遍存在的第一过渡系金属双核酶结 构, 我们有足够的理由相信, 低毒廉价的第一过渡系金 属手性双核催化剂, 将有可能应用到一些长期由贵重金 属 Pt，Pd 或 Rh 催化的反应上，这将带来巨大的经济效 益. 负载型以及非均相可回收循环手性双核催化剂为大 规模工业化提供了可能. 近几年才发展的手性聚合催化 剂, 很有可能推动传统的高分子聚合物产业向手性功能 化方向的革新. 非线性相关试验有助于我们了解催化活 性结构, 为深入研究不对称反应的机理提供参考, 设计 合适的双核催化体系, 更有望在提高催化效能的同时实 现手性放大.

\section{References}

[1] Hino, T.; Matsumoto, Y.; Nagano, S.; Sugimoto, H.; Fukumori, Y.; Murata, T.; Iwata, S.; Shiro, Y. Science 2010, 330, 1666.

[2] Kureshy, R. I.; Khan, N. H.; Sayed, H. R. Tetrahedron: Asymmetry 2001, 12, 433.

[3] Gavrilova, A. L.; Bosnich, B. Chem. Rev. 2004, 104, 349.

[4] Robson, R. Dalton Trans. 2008, 5113.

[5] Thakur, S. S.; Chen, S. W.; Li, W. J.; Shin, C. K.; Kim, S. J.; Koo, Y. M.; Kim, G. J. J. Organomet. Chem. 2006, 691, 1862.

[6] Kureshy, R. I.; Das, A.; Khan, N. H.; Abdi, S. H. R.; Bajaj, H. C. ACS Catal. 2011, 1, 1529.

[7] Wu, Y.-L.; Yao, Z.-J.; Hu, T.-S. Modern Organic Synthesis Chemistry, Science Press, Beijing, 2006, pp. 162 164 (in Chinese). (吴毓林, 姚祝军, 胡泰山, 现代有机合成化学, 科学出版社, 北 京, 2006, pp. 162 164.)

[8] Satyanarayana, T.; Abraham, S.; Kagan, H. B. Angew. Chem., Int. Ed. 2009, 48, 456.

[9] Finn, M. G.; Sharpless, K. B. J. Am. Chem. Soc. 1991, 113, 113.

[10] Noyori, R.; Suga, S.; Oka, H.; Kitamura, M. Chem. Rev. 2001, 1, 85.

[11] Sato, I.; Urabe, H.; Ishiguro, S.; Shibata, T.; Soai, K. Angew. Chem., Int. Ed. 2004, 42, 315.

[12] Zhang, Z. J.; Wan, B. S.; Chen, H. L. Chin. J. Org. Chem. 2003, 23, 636 (in Chinese). (张占金, 万伯顺, 陈惠麟, 有机化学, 2003, 23, 636.)

[13] Gao, Y. H.; Xu, L. W.; Xia, C. G. J. Mol. Catal. 2008, 22, 6 (in Chinese). (高月华, 徐利文, 夏春谷, 分子催化, 2008, 22, 6.)

[14] Arjmand, F.; Parveen, S.; Afzal, M.; Shahid, M. J. Photochem. Photobiol. B 2012, 114, 15.

[15] Howson, S. E.; Scott, P. Dalton Trans. 2011, 40, 10268.

[16] Pardo, E.; Ruiz-Garcia, R.; Lloret, F.; Faus, J.; Julve, M.; Journaux, Y.; Novak, M. A.; Delgado, F. S.; Ruiz-Perez, C. Chem. Eur. J. 2007, 13, 2054.
[17] Iglesias, A. L.; Aguirre, G.; Somanathan, R.; Parra-Hake, M. Polyhedron 2004, 23, 3051.

[18] Lou, B. Y.; Wei, Y. Q.; Lin, Q. CrystEngComm 2012, 14, 2040.

[19] Brunel, J. M. Chem. Rev. 2005, 105, 857.

[20] Belokon, Y. N.; Caveda-Cepas, S.; Green, B.; Ikonnikov, N. S.; Khru-stalev, V. N.; Larichev, V. S.; Moscalenko, M. A.; North, M.; Orizu, C. Tararov, V. I.; Asinazzo, M. T.; Timofeeva G. I.; Yashkina, L. V. J. Am. Chem. Soc. 1999, 121, 3968.

[21] Belokon, Y. N.; Gutnov, A. V.; Moskalenko, M. A.; Yashkina, L. V.; Lesovoy, D. E.; Nicolai S. Ikonnikov, N. S.; Laricheva, V. S.; North, M. Chem. Commun. 2002, 244.

[22] Belokon, Y. N.; Blacker, A. J.; Carta, P.; Clutterbuck, L. A.; North, M. Tetrahedron 2004, 60, 10433.

[23] Zhang, Z. P.; Wang, Z.; Zhang, R. Z.; Ding, K. L. Angew. Chem. 2010, 122, 6898 .

[24] Palomo, C.; Oiarbide, M.; Laso, A. Eur. J. Org. Chem. 2007, 2561.

[25] Bandini, M.; Piccinelli, F.; Tommasi, S.; Umani-Ronchi, A.; Ventrici, C. Chem. Commun. 2007, 616.

[26] Jammi, S.; Saha, P.; Sanyashi, S.; Sakthivel, S.; Punniyamurthy, T. Tetrahedron 2008, 64, 11724.

[27] Shi, Y.; Mao, Z. J.; Xue, Q. C.; Zhu, C. J.; Hu, H. W.; Cheng, Y. X. Inorg. Chem. Commun. 2012, 20, 259.

[28] Trost, B. M.; Yeh, V. S. C.; Ito, H.; Bremeyer, N. Org. Lett. 2002, 4, 2623.

[29] Trost, B. M.; Andrew, H.; Weiss, A. H.; von Wangelin, A. J. Am. Chem. Soc. 2006, 128, 8.

[30] Gommermann, N.; Knochel, P. Chem. Eur. J. 2006, 12, 4380.

[31] Gommermann, N.; Koradin, C.; Polborn, K.; Knochel, P. Angew. Chem., Int. Ed. 2003, 42, 5763.

[32] Muthupandi, P.; Alamsetti, S. K.; Sekar, G. Chem. Commun. 2009, 3288.

[33] Muthupandi, P.; Sekar, G. Org. Biomol. Chem. 2012, 10, 5347.

[34] Au-Yeung, T. L. L.; Chan, S. S.; Chan, A. S. C. Adv. Synth. Catal. 2003, 345, 537.

[35] Hanawa, H.; Uraguchi, D.; Konishi, S.; Hashimoto, T.; Maruoka, K. Chem. Eur. J. 2003, 9, 4405.

[36] Waltz, K. M.; Carroll, P. J.; Walsh, P. J. Organometallics 2004, 23, 127.

[37] Ay, S.; Ziegert, R. E.; Zhang, H.; Nieger, M.; Rissanen, K.; Fink, K.; Kubas, A.; Gschwind, R. M.; Brase, S. J. Am. Chem. Soc. 2010, 132,12899

[38] Lauterwasser, F.; Vanderheiden, S.; Brase, S. Adv. Synth. Catal. 2006, 348, 443.

[39] Chen, Z. H.; Yakura, K.; Matsunaga, S.; Shibasaki, M. Org. Lett. 2008, 10, 3239.

[40] Kato, Y.; Furutachi, M.; Chen, Z.; Mitsunuma, H.; Matsunaga, S.; Shibasaki, M. J. Am. Chem. Soc. 2009, 131, 9168.

[41] Mouri, S.; Chen, Z.; Mitsunuma, H.; Furutachi, M.; Matsunaga, S.; Shibasaki, M. J. Am. Chem. Soc. 2010, 132, 1255.

[42] Mouri, S.; Chen, Z.; Matsunaga, S.; Shibashi, M. Heterocycles 2012, 84, 879 .

[43] Gopinath, P.; Watanabe, T.; Shibasaki, M. Org. Lett. 2012, 14, 1358.

[44] Xu, Y. J.; Matsunaga, S.; Shibasaki, M. Org. Lett. 2010, 12, 3246.

[45] Wezenberg, S. J.; Salassa, G.; Escudero-Adán, E. C.; BenetBuchholz, J.; Kleij, A. W. Angew. Chem., Int. Ed. 2011, 50, 713.

[46] Escárcega-Bobadilla, M. V.; Anselmo, D.; Wezenberg, S. J.; Escudero-Adán, E. C.; Belmonte, M. M.; Martina, E.; Kleij, A. W. Dalton Trans. 2012, 41, 9766.

[47] Cozzi, P. G. Chem. Soc. Rev. 2004, 33, 410

[48] Wezenberg, S. J.; Kleij, A. W. Adv. Synth. Catal. 2010, 352, 85.

[49] Zheng, X. L.; Jones, C. W.; Weck, M. Adv. Synth. Catal. 2008, 350, 
255.

[50] Venkatasubbaiah, K.; Gill, C. S.; Takatani, T.; Sherrill, C. D.; Jones, C. W. Chem. Eur. J. 2009, 15, 3951.

[51] Sadhukhan, A.; Khan, N. H.; Roy, T.; Kureshy, R. I.; Abdi, S. H. R.; Bajaj, H. C. Chem. Eur. J. 2012, 18, 5256.

[52] Haak, R. M.; Castilla, A. M.; Belmonte, M. M.; Escudero-Adán, E. C.; Benet-Buchholz, J.; Kleij, A. W. Dalton Trans. 2011, 40, 3352

[53] Haak, R. M.; Belmonte, M.; Escudero-Adán, E. C.; BenetBuchholz, J.; Kleij, A. W. Dalton Trans. 2010, 39, 593.

[54] Chen, S. W.; Kawthekar, R. B.; Kim, G. J. Tetrahedron Lett. 2007, 48, 297.

[55] Jin, L.; Huang, Y.; Jing, H.; Chang, T.; Yan, P. Tetrahedron: Asymmetry 2008, 19, 1947.

[56] Dro, C.; Bellemin-Laponnaz, S.; Welter, R.; Gade, L. H. Angew. Chem., Int. Ed. 2004, 43, 4479.

[57] Foltz, C.; Stecker, B.; Marconi, G.; Wadepohl, H.; Bellemin-Laponnaz, S.; Gade, L. H. Chem. Commun. 2005, 5115.

[58] Kalow, J. A.; Doyle, A. G. J. Am. Chem. Soc. 2010, 132, 3268.

[59] Kalow, J. A.; Doyle, A. G. J. Am. Chem. Soc. 2011, 133, 16001.

[60] Ballistreri, F. P.; Brinchi, L.; Germani, R.; Savelli, G.; Tomaselli, G. A.; Toscano, R. M. Tetrahedron 2008, 64, 10239.

[61] Sun, Y.; Tang, N.; Liu, X. W.; Liu, W. S. Chin. Chem. Lett. 2004, $15,973$.

[62] Chen, L. H.; Cheng, F. X.; Jia, L.; Zhang, A. J.; Wu, J. C.; Tang, N. Chirality 2011, 23, 69.

[63] Patel, M. J.; Trivedi, B. M. Appl. Organomet. Chem. 2006, 20, 521.

[64] Romakh, V. B.; Therrien, B.; Süss-Fink, G.; Shulpin, G. B. Inorg. Chem. 2007, 46, 1315.

[65] Yeung, H. L.; Sham, K. C.; Tsang, C. S.; Lau, T. C.; Kwong, H. L. Chem. Commun. 2008, 3801.

[66] Sham, K. C.; Yeung, H. L.; Yiu, S. M.; Lau, T. C.; Kwong, H. L. Dalton Trans. 2010, 39, 9469.

[67] Rich, J.; Rodríguez, M.; Romero, I.; Vaquer, L.; Sala, X.; Llobet, M.; Corbella, M.; Collombe, M.; Fontrodona, X. Dalton Trans.
2009, 8117.

[68] Legros, J.; Bolm, C. Chem. Eur. J. 2005, 11, 1086.

[69] Costas, M.; Chen, K.; Que, L. Coord. Chem. Rev. 2000, 200.

[70] Takizawa, S.; Katayama, T.; Kameyama, C.; Onitsuka, K.; SuzuKi, T.; Yanagida, T.; Kawa, T.; Sasai, H. Chem. Commun. 2008, 1810.

[71] Takizawa, S.; Arteaga, F. A.; Yoshida, Y.; Kodera, J.; Nagata, Y.; Sasai, H. Dalton Trans. 2013, DOI: 10.1039/c2dt32202a in press.

[72] Mutti, F. G.; Gullotti, M.; Casella, L.; Santagostini, L.; Pagliarin, R.; Andersson, K. K.; Iozzie, M. F.; Zoppellaro, G. Dalton Trans. 2011, 40, 5436.

[73] Champouret, Y. D. M.; Nodes, W. J.; Scrimshire, J. A.; Singh, K.; Solan, G. A.; Young, I. Dalton Trans. 2007, 4565.

[74] Nakano, K.; Nozaki, K.; Hiyama, T. J. Am. Chem. Soc. 2003, 125, 5501.

[75] Nakano, K.; Hiyama, T.; Nozaki, K. Chem. Commun. 2005, 1871.

[76] Nakano, K.; Hashimotoa, S.; Nozaki, K. Chem. Sci. 2010, 1, 369.

[77] Noda, S.; Nakamura, A.; Kochi, T.; Chung, L. W.; Morokuma, K.; Nozaki, K. J. Am. Chem. Soc. 2009, 131, 14088.

[78] Cohen, C. T.; Coates, G. W. J. Polym. Sci., Part A: Polym. Chem. 2006, 44, 5182.

[79] Thomas, R. M.; Widger, P. C. B.; Ahmed, S. M.; Jeske, R. C.; Hirahata, W.; Lobkovsky, E. B.; Coates, G. W. J. Am. Chem. Soc. 2010, 132, 16520.

[80] Hirahata, W.; Thomas, R. M.; Lobkovsky, E. B.; Coates, G. W. J. Am. Chem. Soc. 2008, 130, 17658.

[81] Otero, A.; Fernández-Baeza, J.; Sánchez-Barba, L. J.; Tejeda, J.; Honrado, M.; Garcés, A.; Lara-Sánchez, A.; Rodríguez, A. M. Organometallics 2012, 31, 4191.

[82] Iglesias, A. L.; Aguirre, G.; Somanathan, R.; Parra-Hake, M. Polyhedron 2004, 23, 3051

[83] Gao, J.; Woolleya, F. R.; Zingaro, R. A. Org. Biomol. Chem. 2005, 3,2126

[84] Zhu, S. F.; Xu, B.; Wang, G. P.; Zhou, Q. L. J. Am. Chem. Soc. 2012, 134, 436 .

(Zhao, C.) 